\title{
A. I. Schtscherbakow: die erste moderne Theorie der Ätiologie und der Pathogenese von Magen- und Zwölffingerdarmgeschwüren
}

\section{Inhaltsverzeichnis}

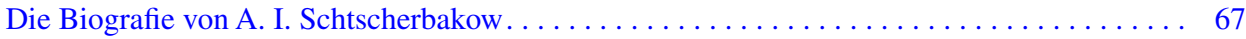

Die Analyse theoretischer Modelle zur Ätiologie und Pathogenese des Magen- und Zwölffingerdarmgeschwürs in der internationalen Wissenschaft des 19. Jahrhunderts . . . . . . . . . . 69 A. I. Schtscherbakows Experiment zur Untersuchung von Magensekretion . . . . . . . . . 81 Zur Theorie über die Entstehung und Entwicklung des Magengeschwürs von A. I. Schtscherbakow . . . . . . . . . . . . . . . . . . . . . . . . . . . . . . . . 91

Die Geschichte der Wissenschaft ist reich an Entdeckern, die der Vergessenheit anheimgefallen sind. Die russische Vorrangstellung bei der Erforschung der Ätiologie und der Pathogenese des Magen- und Zwölffingerdarmgeschwürs ist ein Beispiel für eine Serie vergessener Entdeckungen. Die Schriften gegenwärtiger Therapeuten - Gastroenterologen und Chirurgen -, die sich mit Problemen der Magenchirurgie befassen, bieten dem Leser eine breite Auswahl von Antworten auf die Frage, wer wann die modernen Vorstellungen zu Ätiologie und Pathogenese des Magen- und Zwölffingerdarmgeschwürs formuliert hat. Die Medizin behandelt die Geschwürerkrankung als Folge der Wirkung von aggressivem Mageninhalt auf die Wände von Magen und Zwölffingerdarm; dabei wird die Bedeutung vielfältiger lokaler (Struktur der Schleimhaut, ihre Blutversorgung u.s.w.) und allgemeiner Faktoren (Störungen des Nervensystems) anerkannt. W. Ch. Wassilenko, A. L. Grebnew und A. A. Scheptulin formulierten in ihrer klassischen Monografie „Die Geschwürerkrankung “ diese Auffassung wie folgt:

„Nach den heutigen Vorstellungen wird der Mechanismus der Geschwürbildung sowohl im Magen als auch im Zwölffingerdarm durch die Störung des Zusammenwirkens von aggressivem Magensaft und der eigentlich resistenten Schleimhaut des gastroduodenalen Bereichs ausgelöst. Es handelt sich also um eine Verstärkung des

D. A. Balalykin, Russische Errungenschaften in der Physiologie der Verdauung, 
ersten Faktors (Magensaft) und eine Schwächung des zweiten (Schleimhaut). Dementsprechend werden als ätiologische, d.h. zur Entwicklung der Magenerkrankung prädestinierende Faktoren jene angesehen, die die sauren und enzymatischen Eigenschaften des Mageninhalts entweder verstärken (Erhöhung der Sekretion der Salzsäure und des Pepsins, Störung der motorischen Magen- und Zwölffingerdarmfunktion) oder die Resistenz der Schleimhaut des gastroduodenalen Bereichs schwächen können (Schädigung der Schleimhautbarriere, Unterdrückung der Regenerierungsprozesse der Epithelzellen, Störung des Kreislaufs in der Schleimhaut u.s.w.). Angesichts dieser Erkenntnisse - deren Existenz mehrere experimentelle und klinische Beobachtungen bestätigt haben - wird die ätiologische Rolle von Ernährungsfehlern und schädlichen Gewohnheiten, von eingenommenen Arzneimitteln, psychologischen und neurogenen Faktoren sowie von genetisch bedingten Mechanismen behandelt. "1

Ich möchte hervorheben, dass wir im Rahmen der medizinhistorischen Untersuchung die Entdeckung des Konzepts der Ätiologie und Pathogenese einer Geschwürerkrankung und deren Bedeutung vom heutigen Standpunkt aus diskutieren. Einige Wissenschaftler sprechen die Entdeckung J. Bergmann (1913) zu, andere A. W. Speranski (1930). Manche betonen die Rolle von K. M. Bykow und I. T. Kurzin (1949, 1952). Unter Chirurgen ist die Auffassung verbreitet, dass die These von der aggressiven Magensäure und der geschädigten Schleimhaut A. Schey (1959) zuzuschreiben ist.

Schtscherbakow hat am Ende des 19. Jahrhunderts als Erster in der Welt die Theorie von der Ätiologie und Pathogenese der Geschwürerkrankung formuliert. Um A. I. Schtscherbakows Priorität zu begründen, habe ich die Werke von Schtscherbakow selbst und die berühmte Schrift „Historisches, Kritisches und Positives zur Lehre der Unterleibsaffectionen" von Rudolf Virchow verglichen und analysiert. ${ }^{2}$ In der sowjetischen Geschichtsschreibung hat sich die Vorstellung von R. Virchows Priorität mit Bezug auf diese Arbeit entwickelt. Zu meiner großen Überraschung hat keiner der bekannten sowjetischen (und auch nicht sowjetischen) Chirurgen sowie Spezialisten für Gastroenterologie auf diese Arbeit verwiesen oder ein einziges Fragment davon reproduziert. ${ }^{3}$ Schtscherbakow Vorrangstellung war den russischen Wissenschaftlern am Anfang des 20. Jahrhunderts gut bekannt. Später aber hörte man auf, auf Schtscherbakow zu verweisen; seine Schriften fielen dem Vergessen anheim.

\footnotetext{
${ }^{1}$ Wassilenko, W. Ch., Grebnew, A. L., Scheptulin, A. A.: Die Geschwürerkrankung. Moskau, 1987.

${ }^{2}$ Virchow, R., Historischen, Kritisches und Positives zur Lehre der Unterleibsaffectionen. Virchow's Archiv, 1853.

${ }^{3}$ Diese klassische Arbeit von R. Virchow wurde zuerst ins Russische übersetzt und als Anhang zur russischen Ausgabe dieses Buches veröffentlicht. Balalykin, D. A., Prioritäten der russischen Wissenschaft im Bereich der Physiologieforschung und der experimentellen Magenchirurgie im 19. Und frühen 20. Jh., 2. Aufl., Moskau, 2013. S. 158-224.
} 
Anscheinend hat hier leider die extreme Politisierung der sowjetischen Wissenschaft eine Rolle gespielt. Offensichtlich wurde A. I. Schtscherbakow bei den Historikern der Wissenschaft der frühen Sowjetzeit als ein politischer Feind gesehen - als einer der sogenannten weißen Emigranten, d. h. Wissenschaftler, die die antibolschewistische Bewegung unterstützten und nach Westen emigriert sind. Die Namen von hervorragenden russischen Wissenschaftlern, die eine solche Wahl getroffen haben, sind in Vergessenheit geraten und ihre Leistungen wurden vollständig ignoriert.

Die Wissenschaftshistoriker dürfen keinesfalls die Fortschritte der russischen Medizin in der Sowjetperiode herunterspielen. Jedoch ist es offensichtlich, dass im Bereich der Magenchirurgie die sowjetische Wissenschaft Mitte des 20. Jahrhunderts in vielerlei Hinsicht unter dem Niveau anderer entwickelter Länder anzusiedeln ist. Die wissenschaftliche Kontinuität, die Beziehung zu den Schriften unserer Forscher Ende des 19. bzw. Anfang des 20. Jahrhunderts war unterbrochen. Wir sehen, dass die Leistungen der Physiologie und der experimentellen Magenchirurgie, die mit der Arbeit von I. P. Pawlow und seiner Schule, mit W. F. Dagajew, A. I. Schtscherbakow und anderen verbunden ist, in der Heimat kaum berücksichtigt wurden.

\section{Die Biografie von A. I. Schtscherbakow}

Schtscherbakow wurde 1858 in Moskau geboren. Nach dem Abitur am zweiten Moskauer Gymnasium besuchte er die naturwissenschaftliche Abteilung der physikalisch-mathematischen Fakultät der Kaiserlichen Moskauer Universität. Nach dem Abschluss dort (1879) arbeitete er als Laborant im Labor für organische Chemie bei dem bekannten Chemiker Professor W. W. Markownikow (organische Chemie). Hier schloss Schtscherbakow seine ersten wissenschaftlichen Studien ab, die der Untersuchung der Eigenschaften von Zinkpropyl gewidmet waren. Zugleich nahm Alexej Iwanowitsch das Studium an der medizinischen Fakultät der Moskauer Universität auf (im dritten Studienjahr), das er 1883 abschloss. Von 1885 bis 1887 war er ohne feste Anstellung in der propädeutischen Klinik von Professor M. P. Tscherinow (1874-1905) tätig. Im Labor des Instituts für Allgemeine Pathologie arbeitete er bei Prof. A. B. Focht. Unter dessen Leitung bereitete er seine Dissertation „Über die Bedingungen der Entwicklung des runden Magengeschwürs (Ulcus ventriculi chronicum rotundum) “ vor, die er 1892 erfolgreich beendete. Damals arbeitete er auch als Assistent an der propädeutischen Klinik und zugleich als Privatdozent an der Klinik für Innere Krankheiten.

Laut den Erinnerungen von W. P. Filatow, damals Student an der Moskauer Universität, ,waren die engsten Mitarbeiter Tscherinows die Dozenten A. I. Schtscherbakow und A. I. Predtetschenski. Manchmal haben sie statt Tscherinow vor den Studenten referiert. Sie führten ausgezeichnete praktische Unterrichtsveranstaltungen (über die physikalischen Forschungsmethoden und Analysen) durch. Beide wurden später große Wissenschaftler und Professoren. 14 Jahre später traf ich A. I. Schtscherbakow in Odessa." Alexej Iwanowitsch war nicht nur für Professor Tscherinow eingesprungen, 
sondern trug auch ergänzend zum Hauptkurs der Diagnostik gemeinsam mit dem Privatdozenten N. S. Kischkin und G. N. Gabritschewski den Kurs „Chemische, physikalische und mikroskopische Verfahren zur Untersuchung der inneren Organe “ vor.

Im März 1895 wurde Schtscherbakow zum Professor an der Kaiserlichen Universität Warschau ernannt. Er besetzte den Lehrstuhl der therapeutischen Hospitalklinik, der wegen des Todes seines Leiters Professor Jakow Jakowlewitsch Stolnikow 1894 vakant geworden war. Seinen Kurs eröffnete Schtscherbakow mit dem Vortrag „Über die Aufgaben des klinischen Unterrichts der medizinischen Wissenschaft". In dieser Rede sprach er davon, dass die Hospitalklinik einen besonderen Charakter habe, der sich von dem anderer Kliniken etwas unterscheide. Laut dem damals üblichen Unterrichtsplan besuchten die Studenten die medizinische Klinik drei Jahre lang. Im ersten Jahr wurde in der propädeutischen Klinik hauptsächlich praktische Semiotik - die Lehre von der Ermittlung der Krankheitszeichen bei den Kranken und das Vermögen, die Bedeutung dieser Zeichen einzuschätzen - studiert. In der Fakultätsklinik (viertes Studienjahr) und in der Hospitalklinik (fünftes Studienjahr) wurde der Unterricht im vollen Umfang geführt, den die Pflichten des Arztes gegenüber den Kranken erforderten.

Im Warschau veröffentlichte Schtscherbakow die Monografie „Zur Schlammkur geeignete Gegenden im Europäischen Russland“ (1898). Er veröffentlichte die Aufsätze „Über einige Methoden zur Untersuchung des Stoffwechsels und des Blutes“ (1900) und „Grundriss über die Salzseen in Lyssyje Gory“ (1902).

Von 1903 bis 1905 wirkte er auch als Dekan der medizinischen Fakultät der Universität Warschau. Wegen der Schließung dieser Universität von 1905 bis 1908 wurde er an die Neurussische Universität (Odessa ${ }^{4}$ ) zwecks Leitung des vakanten Lehrstuhls der Hospitalklinik versetzt (Oberste Verordnung vom 28. Juli 1907). Außerdem wirkte er seit dem 19. Januar 1908 als Prorektor der Neurussischen Universität. Nach Warschau kehrte Schtscherbakow nicht mehr zurück. Am 4. Juli 1908 wurde er zum Kurator des Lehrbezirks Odessa ernannt. Am 18. März 1913 wird er in den Lehrbezirk Riga als Kurator versetzt, zu welchem die Gouvernements Kurland, Livland und Estland gehörten. ${ }^{5}$

Während des Bürgerkriegs zog Schtscherbakow wieder nach Odessa, wo er als Privatdozent an der medizinischen Fakultät der Neurussischen Universität tätig war.

1919 wurde in Odessa für einige Monate die Sowjetmacht hergestellt. Mit der Verordnung des Kommissars für Bildungswesen wurde Schtscherbakow ,aus dem Dienst an der Neurussischen Universität entlassen und aus den Listen ihrer Dozenten ausgeschlossen“. Im Juni desselben Jahres wurde er von der außerordentlichen Kommission von Odessa verhaftet, in deren unrühmlich bekannten Kellern er einige sehr schwere Wochen verbrachte.

\footnotetext{
${ }^{4}$ Diese Stadt liegt heute auf dem Staatsgebiet der Ukraine.

${ }^{5}$ Heute ist es das Territorium der unabhängigen EU-Staaten Litauen, Lettland und Estland.
} 
Nach der Besetzung Odessas 1919 durch die Freiwilligenarmee ${ }^{6}$ erhielt er wieder eine Stellung an der Universität. Damals wurde der Lehrstuhl der ärztlichen Diagnostik an der propädeutischen Klinik vakant; der gleiche Lehrstuhl war auch in Odessa im obersten medizinischen Seminar für Frauen frei. Alexej Iwanowitsch wurde mit der Leitung beider Lehrstühle beauftragt.

1919 veröffentlichte er seinen „,Grundriss über den Mineralschlamm im Süden Russlands“. An der Universität und im Seminar arbeitete er auch im Januar des nächsten Jahres, aber Anfang Februar 1920 gelang es ihm, Odessa vor der Rückkehr der Bolschewiken zu verlassen. Nach Russland ging er nicht mehr zurück. Den Rest seines Lebens verbrachte er in Belgrad. In der Emigration arbeitete er erfolgreich; er bekleidete eine Professur an der Universität Belgrad und erreichte viel für die Entwicklung der Klimalehre und der Balneologie in Jugoslawien. ${ }^{7}$

\section{Die Analyse theoretischer Modelle zur Ätiologie und Pathogenese des Magen- und Zwölffingerdarmgeschwürs in der internationalen Wissenschaft des 19. Jahrhunderts}

Wesentliches Charakteristikum der Medizinentwicklung in der zweiten Hälfte des 19. Jahrhunderts war - wie bereits ausführlich dargestellt - die zunehmende Orientierung an den Prinzipien und Erkenntnissen der Naturwissenschaften. Die methodische Grundlage der Forschung von Alexej Iwanowitsch Schtscherbakow zielte darauf ab, mit naturwissenschaftlichen Methoden, ausgehend von der Einsicht in die biologischen Prozesse, eine Analyse des Wesens der Geschwürerkrankung zu erreichen. Als Kliniker und Experimentator kannte er den Stand der Forschung im Bereich jener Probleme, die ihn interessierten, und die Schwierigkeiten, mit denen die Kliniker der damaligen Zeit in ihrer Praxis zu tun hatten.

„Mit Stolz aber haben wir das Recht zu sagen“, schrieb Schtscherbakow, „dass die Aufgabe der heutigen Medizin voll und ganz aus dem Streben besteht, sie auf den Boden der strikten, genauen Wissenschaft zu stellen, sie von dem Einfluss der Person, der persönlichen Fertigkeit, zu befreien. Eine Aufgabe, die vielleicht erst in unendlich entfernter Zukunft realisierbar ist, die uns dennoch als heller Stern erscheint, der unsere Wissenschaft zu wahrem Fortschritt und Gedeihen führt. " ${ }^{\text {8 }}$

\footnotetext{
${ }^{6}$ Die Freiwilligenarmee ist ein operativ-strategischer Zusammenschluss der Weißen Armee (antibolschewistische Armee), der 1918-1920 im Süden Russlands existierte.

${ }^{7}$ Jugoslawien war ein europäischer Staat, der sich im Jahr 2008 aufgelöst hat. Derzeit befinden sich die Staaten Serbien, Kroatien, Slowenien, Nordmazedonien, Montenegro und Bosnien und Herzegowina au $\mathrm{f}$ dem Territorium des ehemaligen Staates.

${ }^{8}$ Schtscherbakow, A. I., Über die Bedingungen der Entwicklung des runden Magengeschwürs (Ulcus ventriculi chronicum rotundum). Moskau, 1891, S. 10.
} 
Er war zutiefst überzeugt, dass die Implementierung der Methode des wissenschaftlichen Experiments in die Untersuchung der klinischen Phänomene die Denkweise des Arztes ändert und dass die Probleme im Verständnis der pathologischen Prozesse und ihrer Ursachen auf eine neue Art und Weise anzugehen sind. Er unterstrich, dass beim Experimentieren Modelle entwickelt werden, die Details und Zusammenhänge der beobachteten Erscheinungen aufdecken - Einzelheiten, die am Krankenbett recht oft dem Blick des Arztes entgehen, sowohl infolge der Unwiederholbarkeit der Erscheinungen als auch aufgrund des Verlaufs der Krankheit in jedem einzelnen Fall.

Der Arzt kann die Reihenfolge der Krankheitssymptome beobachten, ist jedoch nicht in der Lage, nach eigener Vorstellung diese Symptome zu erzeugen und zu modellieren oder den Verlauf pathologischer Prozesse zu ändern. Der Arzt beeinflusst die Krankheit mit seinen therapeutischen Maßnahmen aktiv, soll jedoch die Behandlung in Kenntnis der wahren Ursachen und Mechanismen der Krankheitsentwicklung sowie bestimmter Informationen über das Wesen des pathologischen Prozesses aufbauen.

Zum besseren Verständnis führen wir eine Aussage von Schtscherbakow aus seinem berühmten Vortrag „Über die Aufgaben des klinischen Unterrichts der medizinischen Wissenschaft" vom 17. September 1896 an. Auf dieser Grundlage kann man seine Einsicht in die Bedeutung des Experiments für die Aufdeckung des wahren Wesens pathologischer Prozesse einschätzen:

„Das hergebrachte post hoc, ergo propter hoc, das trotz der allgemeinen theoretischen Diskussion nach wie vor eine so wichtige Rolle während der Beobachtung am Krankenbett spielt, wird bei einem richtig geplanten Experiment fast vollkommen ausgeschlossen. Somit wird die in der Klinik beobachtete Erscheinung, die ins Institut der experimentellen Pathologie übertragen wird, von allen zufälligen Nebeneinwirkungen gereinigt, die es bei einem so komplizierten Beobachtungsobjekt gibt, wie es der menschliche Organismus mit seinen unzähligen individuellen Besonderheiten und einem starken Einfluss des psychischen Bereichs ist. Wenn die auf dem Versuchswege gefundene Tatsache in die Klinik eingebracht wird, können wir leichter mit der Masse komplizierter Erscheinungen klarkommen und die uns bereits bekannten, charakteristischen Merkmale von den unwesentlichen, damit offensichtlich im direkten Zusammenhang stehenden trennen."

Somit baute Schtscherbakow die Analyse der Ätiologie und Pathogenese der Ulkuskrankheit auf den Prinzipien der Untersuchung der klinischen Phänomene im Experiment und der Übertragung der Ergebnisse des Experiments auf das Verständnis des komplizierten klinischen Bildes der jeweiligen Pathologie auf.

In der Zeit, die wir beschreiben, gab es in der internationalen Wissenschaft keine ganzheitliche Vorstellung von den Magenfunktionen in gesundem und krankem Zustand. „Das Unwissen um die krankhaften Störungen der Magenfunktionen erschwerte im hohen Maße das Verständnis des Wesens der pathologischen Prozesse, die sich in diesem Organ abspielen, weil die Grundlage seiner Funktionen eben 
chemische Transformationen darstellen."9 Die fehlende ganzheitliche Vorstellung von den Magenfunktionen bei gesunden und kranken Menschen behinderte die nosologische Identifikation der vielfältigen Erscheinungsformen von Magenerkrankungen. „Bestimmte typische Bilder von Magenbeschwerden konnten nicht einmal die besonders hervorragenden Kliniker definieren: Das Bedürfnis, die Krankheitsprozesse zu systematisieren und sie in den Rahmen einer nosologischen Klassifikation zu setzen, stieß auf vollkommen unüberwindbare Hindernisse. “10 Einer der Umstände, der die richtige nosologische Klassifikation verschiedener Formen der Magenpathologie erschwerte, war eine damals verbreitete Vorstellung von der Natur dieser Erkrankungen, die ausschließlich auf pathologisch-anatomischen Daten gründete. Vielfältige chronische Formen von Magenleiden wurden als morphologische Typen aufgefasst: „Catarrhus“, „Carcinoma“, „Ulcus“, „Dilatatio“. „Wenn man die Gesamtheit der Magenbeschwerden in diesen engen anatomischen Rahmen setzt, mussten die Kliniker unwillkürlich die ganze unendliche Vielfalt von Erscheinungen, die bei den Magenkranken beobachtet wurden, zu den individuellen Besonderheiten des Organismus rechnen. "11

Aus dieser Situation ergaben sich auch die unterentwickelten Methoden der Diagnostik und der Therapie. Das Ansammeln der einzelnen, nicht durch ein einheitliches Modell des pathologischen Prozesses miteinander verbundenen Beobachtungen schuf keine Möglichkeit, eine allgemeine Strategie der Behandlung zu erarbeiten. Darauf weist auch die Tatsache hin, dass Kliniker der damaligen Zeit es für möglich hielten, bei vollkommen gleichen krankhaften Erscheinungen unterschiedliche und mitunter durchaus entgegengesetzte Behandlungsansätze anzuwenden.

Die wahre wissenschaftliche Untersuchung der Magenfunktionen begann mit der Entwicklung des Verfahrens der chemischen Analyse des Mageninhalts und der Untersuchung der im Magen ablaufenden chemischen Reaktionen. „Die Einführung der genauen Forschungsmethoden der Chemie in die Klinik“, führte Schtscherbakow aus, „,bildete endlich eine stabile Grundlage für die strikt wissenschaftliche Bearbeitung der Magenpathologie. Diese Methoden machten die sorgfältige Analyse jedes einzelnen Falls von Magenbeschwerden möglich; die Diagnose und die Therapie der Magenerkrankungen erhielten somit die Möglichkeit, sich nicht von subjektiven, oft recht trügerischen Empfindungen der Kranken, sondern von zweifelsfreien, objektiven Forschungsdaten leiten zu lassen." ${ }^{12}$

\footnotetext{
${ }^{9}$ Schtscherbakow, A. I., Über die Bedingungen der Entwicklung des runden Magengeschwürs (Ulcus ventriculi chronicum rotundum). Moskau, 1891, S. 1.

${ }^{10}$ Schtscherbakow, A. I., Über die Bedingungen der Entwicklung des runden Magengeschwürs (Ulcus ventriculi chronicum rotundum). Moskau, 1891, S. 1.

${ }^{11}$ Schtscherbakow, A. I., Über die Bedingungen der Entwicklung des runden Magengeschwürs (Ulcus ventriculi chronicum rotundum). Moskau, 1891, S. 1.

${ }^{12}$ Schtscherbakow, A. 1., Über die Bedingungen der Entwicklung des runden Magengeschwürs (Ulcus ventriculi chronicum rotundum). Moskau, 1891, S. 2.
} 
Die Untersuchung der Ätiologie und der Pathogenese der Geschwürerkrankung betrachtete er als ein komplexes Problem, an dessen Lösung neben der pathologischen Anatomie auch Physiologie, Chemie und experimentelle Pathologie mitwirken müssten. Ihm war klar, dass die zu dieser Zeit vorliegenden Fakten und theoretischen Verallgemeinerungen nur den Beginn der wissenschaftlichen Erkenntnis zum Wesen der Geschwürerkrankung darstellten. Jedoch gab es Grund genug zu glauben, dass die Wissenschaft auf dem richtigen Wege sei und Methoden gefunden waren, mit denen man unwiderlegbare Tatsachen ermitteln und überprüfbare wissenschaftliche Theorien entwickeln kann. „Die Einführung der Methoden für die Untersuchung des Mageninhalts in die klinische Praxis“, folgerte er, ,hatte keine geringere Bedeutung für den Fortschritt unseres Wissens im Bereich der Magenpathologie als beispielsweise die Erfindung der Perkussion und der Auskultation für die Entwicklung der Lehre von den Brusterkrankungen oder des Laryngoskops für Halserkrankungen."13 Für die mit Abstand wichtigste wissenschaftliche Erkenntnis hielt er die Entdeckung des Zusammenhangs zwischen den Störungen der Magensekretion und dem chronischen runden Magengeschwür.

Seine Untersuchungen zur Ätiologie und Pathogenese der Ulkuskrankheit führte Schtscherbakow im Institut für Pathologie der medizinischen Fakultät der Kaiserlichen Moskauer Universität unter der Leitung von A. B. Focht durch. Der Entwicklung seiner eigenen Schlussfolgerungen ging eine detaillierte Analyse der zu dieser Zeit vorhandenen Modelle zu Ursachen und Entstehung der Geschwürerkrankung voraus. Wir erlauben uns daher, ein ähnliches Vorgehen zu verwenden und die Entwicklungsgeschichte jener Vorstellungen zur Ulkuspathologie zu betrachten, die den Ausarbeitungen von Schtscherbakow vorangegangen sind.

Bis zum Anfang des 19. Jahrhunderts gab es keine Lehre zur Geschwürerkrankung als besondere nosologische Einheit. Die antiken Ärzte Hippokrates und Galen hatten lediglich einige Einzelheiten zur Geschwürbildung in der Magenwand beschrieben.

Im 18. Jahrhundert publizierten einige Autoren Kasuistiken zu Magenperforationen, zu Fistelbildungen, zur Verheilung des Geschwürs mit Narbenbildung und zur spezifischen Veränderung der Organform, d. h. zum Verwachsen des Geschwürs mit dem umliegenden Gewebe. In einem Text von Morgagni kann man beispielsweise eine recht ausführliche pathologisch-anatomische Beschreibung des runden Magengeschwürs finden.

Am Ende des 18. und zu Beginn des 19. Jahrhunderts wurden erste Versuche unternommen, vereinzelte Informationen bezüglich der Ulkusbildung im Magen zu einem einheitlichen Gesamtbild zusammenzuführen (Baille 1798; Voigtei 1804). Ein Beweis für das totale Durcheinander bei der Frage über die wesentlichen Merkmale des

\footnotetext{
${ }^{13}$ Schtscherbakow, A. I., Über die Bedingungen der Entwicklung des runden Magengeschwürs (Ulcus ventriculi chronicum rotundum). S. 2-3.
} 
Magengeschwürs stellt der Beitrag von Abercrombie dar, in dem der Autor das Magengeschwür falsch beschrieben und es mit Krebs verwechselt hat.

Die eigentliche Lehre über das runde Magengeschwür entstand, als Cruveilhier es als eine eigenständige nosologische Einheit in seinen von 1829 bis 1835 veröffentlichten Werken definierte. Er betrachtete das Ulkusleiden als eine gesonderte Krankheit, die zu Lebzeiten des Patienten zu diagnostizieren und auf die therapeutisch einzuwirken sei. Cruveilhier führte die nosologische Bezeichnung ,einfaches chronisches Magengeschwür" ein. ${ }^{14}$

Das pathologisch-anatomische Bild des runden Magengeschwürs stellte Cruveilhier mithilfe hervorragender Zeichnungen vor. Ausgehend von seinen eigenen Beobachtungen schilderte Cruveilhier auch das klinische Krankheitsbild recht genau und sehr deutlich. Er beschrieb auch bekannte Verfahren für die Behandlung der Erkrankung. Die Verknüpfung der pathologischen Veränderungen mit dem klinischen Bild war die Grundlage für die Definition des runden Magengeschwürs als einer eigenständigen nosologischen Einheit. Dabei meinte Cruveilhier, dass ihm keine spezifische Ätiologie zugrunde liege. Das Geschwür selbst sei eine Art sekundäre Bildung, die sich auf der Grundlage einer Erosion entwickele, die infolge des pathologischen Prozesses entstehe; diesen bezeichnete bereits Hunter als „Inflammation Ulcerose“. Die zunächst entstandene Erosion verwandle sich infolge bestimmter Umstände in ein Magengeschwür. Für Cruveilhier hatte die Geschwürerkrankung keine definierte Ätiologie, weil sie aus seiner Sicht durch jene Ursache ausgelöst wurde, die eine Gastritis verursacht. Für ihn blieb es ein Rätsel, warum das Geschwür besonders oft bestimmte Magenabschnitte befällt.

Überhaupt mangelte es in der Medizin der damaligen Zeit an einer ganzheitlichen Lehre von den Verdauungsstörungen als einem funktionalen Komplex. Im Wortschatz der Ärzte fehlte sogar der Begriff „Dyspepsie“. Die Frage nach den Störungen des Verdauungschemismus bzw. nach den Störungen der Resorption wurde nicht aufgeworfen. Folglich war der größte Fortschritt bei den Vorstellungen über die Geschwürerkrankung mit den Leistungen der Pathologen verbunden. Versuche eines funktionalen Ansatzes, die mit der Implementierung der Ideen eines „,chronischen Experiments“ zusammenhängen, wurden im internationalen wissenschaftlichen Denken nicht unternommen.

Den nächsten bedeutsamen Schritt bei der Erforschung der Geschwürerkrankung unternahm Rokitansky, dessen Schriften zu diesem Thema aus den Jahren 1834 bis 1842 stammen.

Das runde Magengeschwür bezeichnete er wegen dessen Eigenschaft, in die Tiefe der Magenwand einzudringen, als ,perforierendes“. Er betrachtete das Ulcus ventriculi als eine eigenständige nosologische Einheit und meinte, dass es sich aufgrund seiner Pathogenese von allen anderen im Magen auftretenden Geschwürprozessen unterscheide. Der Entstehung des Ulkus liegt nach Rokitansky die hämorrhagische Erosion zugrunde. Die

\footnotetext{
${ }^{14}$ Cruveilhier, J., Anatomie pathologique du corps humain, Bd. 1, Paris, 1829-1835. Livr. 10.
} 
Erosion selbst entwickelt sich infolge des veränderten Sekrets der Pepsindrüsen, das mit einer Hyperämie verbunden ist. Als Folge dieser Vorgänge wird die Schleimhaut in einigen Abschnitten des Magens ,zerfressen“; anschließend kommt es zur Hämorrhagie. Hämorrhagische Erosionen können verheilen, aber es kann auch rasch ein Geschwür entstehen, falls die Erosion einen größeren Raum einnimmt; insbesondere dann, wenn die Schädigung tief in die Schleimhaut eindringt. Das Ulkus entwickelt sich also aus dem streng abgegrenzten Herd der Hyperämie oder aus dem begrenzten Abschnitt der Schleimhautnekrose und verwandelt sich in Schorf, wobei als Ausgangspunkt die Bildung einer ,normalen“ hämorrhagischen Erosion gesehen wird. ${ }^{15}$

Eine Reihe von Autoren versuchte, die Entstehung des Geschwürs durch den Einfluss verschiedener psychologischer bzw. nervöser Faktoren zu erklären. 1845 zeigte Schiff auf der Grundlage seiner Untersuchungen, dass partielle hämorrhagische Infiltrate und das Erweichen der Magenwand auch ohne direkte Einwirkung auf die Schleimhaut zustande kommen können. Diese Veränderungen treten als Folge einer Störung bestimmter Strukturen des Nervensystems auf, die mit der Innervation des Magens zusammenhängen.

In seinen Experimenten mit Kaninchen durchschnitt Schiff den Thalamus opticus und den Pedunculus cerebri auf einer Seite. Infolgedessen traten innerhalb von vier Tagen hämorrhagische Infiltrationen und Erweichungen der Magenwand ein. In vielen Experimenten erreichte dieser Wissenschaftler die Entwicklung einer Schleimhauterosion, manchmal auch eines perforierten Ulcus ventriculi. Als Ursache dieser Erscheinungen nahm Schiff Schädigungen der zentralen Bahnen der vasomotorischen Magennerven an. Diese Ideen wurde später von Ebstein und Vulpian weiterentwickelt. Die Liste der Strukturen des zentralen Nervensystems, deren Zerstörung eine Geschwürbildung verursachen kann, wurde wesentlich erweitert. ${ }^{16}$ Die Ergebnisse von Schiffs Experimenten beachtete auch Pawlow bei seinen genialen Versuchen zur Untersuchung der Nervenversorgung des Magens, die mit der Entwicklung des experimentellen Modells der Vagotomieoperation abgeschlossen wurden.

Mitte des 19. Jahrhunderts wurde die fälschliche Auffassung der Geschwürerkrankung als eines ,normalen“ atonischen Ulkus verbreitet, das sich infolge mehrerer unspezifischer Ursachen entwickelt (Lebert, 1858; Merkel, 1866).

Im Jahr 1853 veröffentlichte Virchow, wie bereits erwähnt, sein grundlegendes Werk „Historisches, Kritisches und Positives zur Lehre der Unterleibsaffectionen“, in welchem er seine Theorie zur Entstehung der Geschwürerkrankung darlegte. Viele

\footnotetext{
${ }^{15}$ Rokitansky, C., Lehrbuch der pathologischen Anatomie, 3. Aufl., Wien, 1855-1861; 3, S. 170 ff. (1. Aufl. 1842).

${ }^{16}$ Ebstein, W., Experimentelle Untersuchungen über das Zustandekommen von Blutextravasaten in der Magenschleimhaut. Arch. f. exper. Pathol. und Pharm. 1874; Schiff, M., Leçons sur la physiologie de la digestion. Florence et Turins. Paris; Berlin, 1867; Vulpian, A. Leçons sur l'appareil vasomoteur. Paris, 1875.
} 
Autoren richteten bei der Analyse seiner Auffassung ihr Augenmerk nur auf einen Aspekt und behaupteten, er hätte bei der Formulierung seiner Theorie ausschließlich auf die Störungen der Zirkulation als pathologischen Faktor hingewiesen. Tatsächlich war seine Betrachtungsweise der Ätiopathogenese des Ulkus wesentlich tiefgehender. Virchow betonte, dass auf die Schleimhaut des Magens sowohl der Magensaft als auch die infolge der Gärprozesse neu entstandenen Säuren einwirken können. In einem gesunden Magen sei die Wahrscheinlichkeit, dass diese Säuren eine „zerfressende“ Wirkung entfalten, allerdings recht gering. Ein Reizzustand der Magenschleimhaut sei bei Kranken recht oft zu beobachten, nicht jedoch in der Art, dass sie eine Gastromalazie verursachten. ${ }^{17}$

Entsprechend Virchows Vorstellungen nimmt bei beträchtlichen Reizzuständen auch die Schleimsekretion zu, die die innere Oberfläche des Organs schützt. Wenn einer der Reizstoffe in die Magenwand eindringt, wird dieser durch die alkalische Reaktion des Blutes aus den intramuralen Gefäßen heraus neutralisiert. Bei noch stärkeren Reizungen werden aggressive Stoffe durch den Brechreflex aus dem Organ entfernt. Aus Virchows Sicht kann sich die Relation zwischen den ,zerfressenden“ Stoffen und der Schleimhaut ändern, wenn die Magenoberfläche aufhört, eine geschlossene sezernierende Haut zu sein. Das Gleichgewicht zwischen der zerstörenden Wirkung der Reizstoffe und den Schutzkräften des Organismus kann beträchtlich gestört werden, wenn infolge eines Hindernisses die Entleerung von saurem Mageninhalt erschwert ist und das aggressive Medium längere Zeit auf die beschädigten oder nekrotischen Abschnitte der Schleimhautoberfläche einwirkt. Eben deswegen kommt die Ulkusbildung zustande. Aber allein die mengenmäßige Steigerung der Sekretion des Magensaftes kann ohne weitere Voraussetzungen das „Zerfressen“ der Magenwand nicht auslösen. Gäbe es diesen Prozess, müsste man eine großflächige Geschwürbildung im Magen erwarten, was aber nicht zu beobachten ist. Das Ulkus ist immer in einem bestimmten Magenteil lokalisiert, was eindeutig auf die lokalen Faktoren hinweist, die für seine Entwicklung notwendig sind.

Virchow betonte also, dass für die Erklärung der Geschwürentstehung lokale Ursachen zu beachten sind: Die Schleimhaut muss in dem Abschnitt, der ,zerfressen“ wird, vorher verändert sein; dies hängt wiederum von den Zirkulationsbedingungen ab. Die Störungen der Blutversorgung des Magengewebes können auf verschiedenen Wegen und aus unterschiedlichen Gründen entstehen. Der wichtigste Grund läuft nach Virchow auf die hämorrhagische Nekrose der Magenschleimhaut hinaus. Wenn in einem Magenabschnitt die normale Blutzirkulation sistiert, wird dort die Säure nicht durch die alkalisch wirkende Blutreaktion neutralisiert. Dadurch tritt die Geschwürbildung ein. Bei einer Zirkulationsstörung in der Magenmukosa kann auch die normale Magensaftmenge die Ulkusbildung verursachen. Virchow nahm an, dass besonders häufige Ursachen für Zirkulationsstörungen die hämorrhagischen Infiltrationen der

\footnotetext{
${ }^{17}$ Virchow, R., Historisches, Kritisches und Positives zur Lehre der Unterleibsaffectionen. Virchow's Archiv, 1853.
} 
Magenwand sind, welche infolge der Erkrankung der Arterien, ihrer Verstopfung und des nachfolgenden Blutstaus, seltener aber infolge der Kontraktion der Muskelschicht eintreten. Er ließ auch den Einfluss einer Kreislaufstörung im Bereich der Vena portae mit Erweiterung der Magenvenen und Entwicklung einer Hyperämie der Mukosa zu, was zur hämorrhagischen Nekrose und konsekutiv zur Entwicklung des chronischen Geschwürs prädestiniert. Akute und chronische Katarrhe und vor allem jene Formen, die mit starkem Erbrechen einhergehen, können auch ohne Blutstau in der Pfortader eine Hyperämie der Schleimhaut, hämorrhagische Erosionen und hämorrhagische Ulzera auslösen. Sowohl Erkrankungen der Gefäßwände, Aneurysmen und variköse Erweiterungen, die sich infolge der Störung der Blutversorgung der Gefäßwände entwickeln, als auch die Einengung des Arterienvolumens und sonstige Gefäßveränderungen verursachen in Verbindung mit dem Säurefaktor die Ulkusbildung. Aber nicht alle Geschwürbildungen entwickeln sich zu Magengeschwüren; sehr oft verheilen sie. Dafür verantwortlich ist nach Virchow die Wiederherstellung der normalen Zirkulationsverhältnisse auf der Oberfläche des Geschwürdefekts. Dadurch wird die Salzsäure mit dem Alkali des Blutes neutralisiert: Auf der Geschwüroberfläche entsteht dann eine Art Schutzfolie aus den Produkten der chemischen Reaktion.

Beim Aufbau seines Modells zur Ätiologie und Pathogenese der Geschwürerkrankung berücksichtigte Virchow die Ergebnisse der Untersuchungen anderer Wissenschaftler. Beispielsweise schrieb Morin bereits im Jahr 1800 über Veränderungen bei der Zirkulation und der Versorgung der Magenwand, die zur Geschwürbildung führen können. Gleichzeitig kann man nicht behaupten, dass Virchow eine vollständige Theorie der Ursachen und der Entstehung der Geschwürerkrankung formulierte. Der Grund dafür war wiederum seine methodische Einengung. Während der Arbeit mit dem Sektionsmaterial und der Beobachtung des Phänomens der Gastromalazie bei der Bearbeitung des Magenpräparats mit Säure äußerte er die Vermutung, es könnten ,gewisse Schutzfaktoren“ zum Funktionserhalt des gesunden Magens existieren, die eine schädigende Wirkung seines aggressiven Inhalts verhinderten. Diese Annahme ist durchaus naheliegend, wenn man die Tatsache berücksichtigt, dass das Phänomen der Erweichung unter normalen Bedingungen nicht eintritt - trotz des sauren Mageninhalts. Aber ohne experimentelle Bestätigung hatte diese Annahme bloß empirischen Charakter. Vorweggreifend muss man betonen, dass ein solches Experiment zum ersten Mal von Alexej Iwanowitsch Schtscherbakow durchgeführt wurde.

Die Ideen und Forschungen von Virchow bestimmten für lange Zeit das wissenschaftliche Denken auf diesem Gebiet. Die meisten Forscher beachteten bis in die 70er und 80er Jahre des 19. Jahrhunderts ausschließlich einen Aspekt der Erklärungen der Geschwürbildung durch Virchow, nämlich die Stärkung der Widerstandsfähigkeit des Magengewebes: „Leider“, bemerkte Schtscherbakow, „haben die meisten Autoren die von dem Wissenschaftler gestellte Aufgabe eingeengt und richteten das Hauptaugenmerk auf die Untersuchung der Resistenz des Gewebes der Magenwand abhängig von den Zirkulationsveränderungen und ließen die Frage hinsichtlich des Einflusses der Azidität 
des Magensaftes wie auch hinsichtlich der Ursachen des fortschreitenden Prozesses generell beiseite.“

Dieser Ansatz führte die Forscher in eine Sackgasse und drängte weitere Untersuchungen zur Chemie der Magenverdauung und zur Zusammensetzung des Magensaftes als aggressivem Faktor ins Abseits. Infolgedessen entwickelten die Wissenschaftler der damaligen Zeit keine weitergehenden Fragestellungen zum fortschreitenden Verlauf der Geschwürerkrankung und zur pathophysiologischen Begründung einer Behandlungsstrategie.

Virchow hatte recht detailliert die Formen der Gefäßveränderungen beschrieben, die eine Beeinträchtigung der Blutversorgung des Magens verursachen und seine Resistenz mindern. Andere Wissenschaftler der damaligen Zeit intensivierten diese Forschungen zwecks Aufbau theoretischer Modelle zur Ätiopathogenese des Ulkus. Lebert und Sangalli betonten die Rolle der Thrombosen und Embolien der den Magen versorgenden Gefäße bei der Entwicklung eines Ulkus. Die in dieser Zeit berühmten Kliniker und Pathologen, zum Beispiel von Recklinghausen ${ }^{18}$ und Merkel ${ }^{19}$, betrachteten die Geschwürerkrankung als Folge hämorrhagischer Infiltrate der Magenwand, die durch eine Menge von unspezifischen Faktoren (Traumata, Embolien, Arteriosklerose) bedingt sind.

Eine große Rolle in der Pathogenese der Ulkuskrankheit spielten die Schriften von Panum. Ausgehend von Virchows Gedanken führte er Experimente durch, in denen er die Entstehung des Geschwürs infolge einer lokalen Störung der Blutversorgung der Magenwand modellierte. Er löste sie durch die Injektion einer Emulsion aus Wachskugeln aus, mithilfe der retrograden Einführung eines Katheters über die Arteria cruralis bis tief in die Bauchaorta hinein. Bei diesen Experimenten starben die Versuchshunde im Regelfall einige Stunden nach der Injektion der Emulsion infolge einer generalisierten Embolie und konsekutiven Infarkten im Bereich mehrerer Organe. Der „Geschwüreffekt“ wurde am Magenpräparat bei der Obduktion festgestellt. Diese Versuche von Panum zeigen, wie weit die Wissenschaftler der damaligen Zeit von der Klärung des realen Pathomechanismus entfernt waren. Zugleich war es das erste experimentelle, allerdings nicht ganz adäquate Modell für die Herbeiführung lokaler Magenschleimhautveränderungen aufgrund einer Störung der Blutversorgung. ${ }^{20}$

\footnotetext{
${ }^{18}$ Recklinghausen, F. v., Auserlesene pathologisch-anatomische Beobachtungen (embolische Heerde des Magens). Virchow's Archiv, 1864.

${ }^{19}$ Merkel, G., Kasuistischer Beitrag zur Entstehung des runden Magen- und Duodenalgeschwüres, Wien. Med. Presse. 1866, Nr. 30-31; Merkel, G., Ueber einen Fall von chronischem Magengeschwür, Wien. Med. Presse. 1866, Nr. 42-43.

${ }^{20}$ Panum, P. L., Experimentelle Beitraege zur Lehre von der Embolie, Virchow's Archiv, 1862; Panum, P. L., Pepsin und Magenfistelanlegung. Jahrsber. D. Thierchemie. 1871 (ref. Nordisk. Medicinsk. Arkiv. 1871. Bd. 3. H. 2. Nr.9).
} 
Die Rolle embolischer Faktoren wurde von Wissenschaftlern wie Klebs, Axel, Key und Rindfleisch etwas zurückhaltender eingeschätzt, wobei sie auf den vorwiegend lokalen Charakter der Zirkulationsstörungen bei Geschwüren hinwiesen. Klebs konnte sich jedoch die Bildung eines Geschwürs ohne Kreislaufveränderungen kaum vorstellen. Als Ursache dieser Veränderungen erkannte er einen Gefäßkrampf, der zu einer Perfusionsstörung in einem Abschnitt der Magenwand führen konnte. Dieser war im Anschluss der korrodierenden Wirkung des Magensaftes ausgesetzt. ${ }^{21}$

Alle Autoren konnten den umfassenden Charakter der Geschwürkrankheit nicht erfassen, weil sie insbesondere die mögliche Entstehung von Ulcera ventriculi aus hämorrhagischen Erosionen ablehnten. Dabei ließen diese Wissenschaftler die Bedeutung der Wirkung des Magensaftes auf die Wand des Organs praktisch vollständig außer Acht.

Zwischen 1860 und 1890 verfielen einige Forscher auf den Gedanken, Magengeschwüre könnten durch eine Infektion ausgelöst werden (Lebert, Letulle). Dabei erzeugten sie jedoch einen insgesamt kritischen Zustand des Organismus, der schlussendlich zum Magengeschwür führte. Lebert beispielsweise spritzte in die Drosselvene eines Kaninchens Eiter ein, der eine Sepsis auslöste; bei der Obduktion wurde ein Magengeschwür festgestellt. ${ }^{22}$

Durch die Analyse der Literatur kam Schtscherbakow zum Ergebnis, dass kein Autor die konkrete Frage nach jenen Faktoren, die die Spezifität des Magengeschwürs bedingen, beantwortet. Das Gleiche gilt für das Problem seiner unaufhaltsamen Progredienz - auch unter für die Heilung durch Vernarbung günstigen Voraussetzungen.

Um die Mitte des 19. Jahrhunderts machten die Wissenschaftler auch keinen prinzipiellen Unterschied zwischen der Entwicklung eines reaktiven Defekts der Magenschleimhaut auf der einen und der Geschwürkrankheit als Erkrankung mit einer spezifischen Ätiologie bzw. Pathogenese auf der anderen Seite. Sie sahen keinen Zusammenhang zwischen dem klinischen Symptom des Schmerzes, den der Kranke empfand, und den spezifischen Faktoren, welche die progressiven Veränderungen in der Magenwand bewirkten.

Der nächste wichtige Schritt bei der Erforschung der Ulkuskrankheit war der Nachweis, dass das Magengeschwür als nosologische Einheit mit dem Geschwürdefekt der Schleimhaut als spezifischem morphologischem Syndrom nicht identisch ist. Diese Leistung vollbrachte Cohnheim in hervorragender Weise. Schtscherbakow bemerkte

\footnotetext{
${ }^{21}$ Key, A., Om det korrosia magsarets uppkomst. Hygiea. 1870 (ref. Virch.-Hirsch. Jahresb. 1870; 2:155); Klebs, E., Anleitung für die pathologische Anatomie, übers. unter Redakt. von Prof. Rudnew. Sankt Petersburg; 1871 (d. Ausg. 1869); Rindfleisch, E., Lehrbuch der pathologischen Gewebelehre. 4. Aufl. Leipzig, 1875.

${ }^{22}$ Lebert, H. Bericht über die klinisch-medizinische Abtheilung des Züricher Krankenhauses in den Jahren 1855 und 1856, Virchow's Archiv 1858; Lebert, H., Beitrage zur Geschichte und Aetiologie des Magengeschwürs, Berl. Klin. Wochensch. 1876; Letulle, M., Origine infectieuse de certains ulcères simples de l'estomac ou duodenum. Comp. Rend. 1888.
} 
dazu, dass ,die Versuche dieses talentierten Experimentators als Ausgangspunkt für die Entwicklung der modernen Lehre über das Ulcus ventriculi gelten sollten. “23

Cohnheim überschritt den Rahmen eines „akuten“ Versuchs und einer ausführlichen Beschreibung des pathologischen Anschauungsmaterials, indem er sich vornahm, einen fortschreitenden bzw. schleppenden Verlauf der Geschwürkrankheit experimentell so zu modellieren, wie ihn Kliniker bei ihren Patienten beobachteten.

Während seiner Tierversuche regte Cohnheim zunächst die Bildung eines akuten Geschwürs an. Im Unterschied zu seinen Vorgängern erreichte er dies durch eine lokale Embolie, ausgelöst durch eine in eine der Arteriae gastricae breves injizierte Bleichromatemulsion. Bei allen kurze Zeit später getöteten Tieren wurden Magengeschwüre mit großen, klar sichtbaren Rändern und mit einem „reinen“ Boden festgestellt. Wenn ein Tier die zweite Woche nach der Injektion doch überlebte, hatten sich statt eines großen Geschwürs mehrere kleine Ulzera im Magenpräparat gebildet. Nach Ablauf der dritten Woche nach der Injektion wurde eine völlig verheilte und glatte Schleimhaut im Magen der Versuchstiere festgestellt. ${ }^{24}$

Cohnheim verglich den fortschreitenden, nicht selten saisonbedingten zyklischen Charakter des Ulkusleidens, wie ihn die Kliniker kannten, mit den Ergebnissen seiner Versuche und wies darauf hin, dass die Geschwürkrankheit als spezifische nosologische Einheit mit der einfachen Geschwürbildung der Magenschleimhaut, welche durch die Wirkung verschiedener Faktoren entsteht und oft einer schnellen Remission unterliegt, nicht identisch war. Weiterhin unterschied er zwischen dem Entstehungsmechanismus der eigentlichen Geschwürbildung in der Mukosa und dem fortschreitenden, chronisch werdenden Verlauf dieser Geschwürbildung. Durch die Ergebnisse seiner Versuche wurde die Theorie widerlegt, nach der das Ulkusleiden nur auf Zirkulationsstörungen zurückzuführen war. Er postulierte auch, dass es im Magen der Ulkuskranken einen unbekannten, starken Faktor gibt, der in Verbindung mit den Kreislaufstörungen der Magenwand die Geschwürkrankheit eigentlich verursacht. Schtscherbakow resümierte später: Indem Cohnheim die vorhandenen mangelnden Kenntnisse über das Ulcus ventriculi erkannt habe, habe er unvermeidlich die Annahme befördert, dass es im Magen der Geschwürkranken außer den Zirkulationsstörungen, die die Zerstörung des ganzen Organs verursachen, noch etwas Unbekanntes, die Heilung dieser Störung Hemmendes gibt. Cohnheim ließ die Frage offen, ob dieses Unbekannte aus einer nicht normalen Vermehrung des Magensaftes bestehe, da es dazu keine positiven Daten gebe. ${ }^{25}$

\footnotetext{
${ }^{23}$ Schtscherbakow, A. I., Über die Bedingungen der Entwicklung des runden Magengeschwürs (Ulcus ventriculi chronicum rotundum), S. 289.

${ }^{24}$ Cohnheim, J. F., Allgemeine Pathologie: in Bd. Übersetzung. W. Ssigrisst, Bd. 2, Sankt Petersburg, 1881.

${ }^{25}$ Schtscherbakow, A. I., Über die Bedingungen der Entwicklung des runden Magengeschwürs (Ulcus ventriculi chronicum rotundum), S. 289.
} 
Nach der Studie Cohnheims war eine große Unsicherheit in Bezug auf die Ätiologie bzw. Pathogenese der Geschwürkrankheit zu verzeichnen. Schtscherbakow konstatierte, dass ,diese Verwirrung erst dann allmählich gelöst wurde, als der Zusammenhang von Ulcus ventriculi und Hypersekretion festgestellt wurde. “26

Die Rolle der verstärkten Magensaftsekretion und der erhöhten Azidität wurde erst um die Mitte der 1880er Jahre ein intensiver beachteter Gegenstand der Forschung. Bei seinen Untersuchungen bezog sich Schtscherbakow vor allem auf Studien von Riegel, Reichman, Jaworski und anderen Forschern. ${ }^{27}$

Riegel entwickelte ein Modell der Ulkuspathogenese, nach welchem zuerst ein vermehrter Säuregehalt konstatiert wurde und danach geschwürige Veränderungen zu beobachten waren. Der erhöhte Säuregehalt verstärkt die Prädisposition zum Magengeschwür, weil sich dadurch sogar unwesentliche Läsionen der Schleimhaut vergrößern und sich allmählich in tiefer reichende Geschwüre umwandeln können. Eben in diesem erhöhten Säuregehalt und nicht in einem anderen Faktor sollte man die Ursache für eine schwierige Heilung des Ulcus chronicum rotundum erkennen, so meinte Riegel.

Viele Wissenschaftler betrachteten die Entstehung und die Entwicklung eines Magengeschwürs als Einzelfall der durch die Einwirkung aggressiver Faktoren ausgelösten Selbstverdauung der Magenwand. Diese Frage wurde unter dem Aspekt einer gestörten Balance von Widerstandsfähigkeit des Magengewebes einerseits und Verdauungsfaktoren andererseits formuliert. 1772 hatte Hunter die Hypothese aufgestellt, nach der die Selbstverdauung (Autolyse) des Magengewebes nicht durch das Vorhandensein eines rudimentären Lebensprinzips (,living principle“) verursacht werde. C. Bernard meinte, dass die Autolyse des Magengewebes nicht erfolgte, weil sich das Magenepithel schnell regenerieren könne. Versuche von Quincke zeigten, dass die bei Anämie eintretenden Gegebenheiten die Heilung der Magengeschwürbildung hemmen.

Pavy, der die Versuche von C. Bernard wiederholt hatte, bekräftigte, dass die Selbstverdauung der Magenschleimhaut durch den Mangel an Substanzen im Blut, welche die Magensaftsäuren neutralisieren, verursacht werde. „Somit hielten wir es für notwendig“, so Schtscherbakow, „uns der Ansicht von Pavy anzuschließen, der einem normalen Kreislauf als Faktor, der die Magenwand gegen die Selbstverdauung schützen soll, postuliert hat. “28 Er hob dabei hervor, dass Pavy allein die Neutralisationsfähigkeit

${ }^{26}$ Schtscherbakow, A. I., Über das runde Magengeschwür, Moskau, Russkaja tipografija, 1888. S. 290.

${ }^{27}$ Jaworski, W., Magenaspirator, zugleich continuirlicher Magen-Irrigationsapparat in Verbindung mit der Sonde ,à double courant“. Deut. Arch. f. klin. Med. 1883; Jaworski, W., Beobachtungen über das Schwinden der Salzsäurereaction und den Verlauf der catarrhalischen Magenerkrankungen. Münch. Med. Wochenschr., 1887; Jaworski, W., Ueber den Zusammenhang zwischen subjectiven Magensymptomen und den objectiven Befunden bei Magenfunctionsstörungen, Wiener Med. Wochenschr. 1886.

${ }^{28}$ Schtscherbakow, A. I., Über die Bedingungen der Entwicklung des runden Magengeschwürs (Ulcus ventriculi chronicum rotundum), S. 316. 
des Blutes als Schutzmechanismus betonte. Eigene Versuche von Schtscherbakow zeigten, dass es im Blut auch andere Faktoren, die die Magenwand vor Selbstverdauung schützen, gibt.

Vorbereitende Arbeiten zur Erstellung seiner eigenen Theorie über das Auftreten und die Entwicklung von Magengeschwüren wurden von A.I. Schtscherbakow nicht nur im Hinblick auf eine kritische Analyse der damals bereits existierenden Ideen und Fakten, auf deren Grundlage Modelle der Ätiologie und Pathogenese von Geschwüren erstellt worden waren, durchgeführt. Er hatte auch die Geschichte der Entwicklung von Methoden zur Untersuchung der Funktionen des Magens und der Verdauungsmechanismen genau studiert. Umfassendes Wissen und Kritik an der damals existierenden experimentellen Methodik ermöglichten es A.I. Schtscherbakow, seine bahnbrechende Forschung erfolgreich abzuschließen.

\section{A. I. Schtscherbakows Experiment zur Untersuchung von Magensekretion}

Als er frühere Studien über die sekretorische Aktivität des Magens und seiner Rolle bei der Verdauung besprach, erklärte A.I. Schtscherbakow: „Bis zur Mitte des letzten Jahrhunderts war das Konzept des ,Magensaftes' in dem Sinne, wie es derzeit allgemein akzeptiert wird, von Naturwissenschaftlern noch nicht etabliert worden. Die sekretorische Funktion des Magens wurde damals nicht als notwendiges Element der peptischen Aktivität des Organs angesehen.“29

Es brauchte annährend zwei Jahrhunderte, um die Rolle des Magensaftes als wichtigsten Verdauungsfaktor in der Welt der Wissenschaft zu verstehen. Grundsätzlich wurden wichtige Entdeckungen gemacht, die A.I. Schtscherbakow in seinen Arbeiten detailliert analysierte und zeigte, wie sich moderne Vorstellungen von der Rolle des Magensaftes bei normalen und pathologischen Funktionszuständen dieses Organs entwickelten.

Seiner Meinung nach gehen die ersten Versuche einer wissenschaftlichen Untersuchung der Magensekretion auf die erste Hälfte des 16. Jahrhunderts zurück und wurden von de Réaumur und Spallanzani durchgeführt. Den Studien dieser Wissenschaftler ging die Arbeit von Vertretern der Accademia del Cimento voraus, die 1667 die motorischen Funktionen des Magens bei Vögeln untersuchten. Ihre Versuche wurden aufgrund der unzureichend entwickelten experimentellen Technik und des folglich empirischen Charakters der Schlussfolgerungen nicht zu einem Ausgangspunkt für die Untersuchung der Magenfunktion.

\footnotetext{
${ }^{29}$ Schtscherbakow, A. I., Über die Bedingungen der Entwicklung des runden Magengeschwürs (Ulcus ventriculi chronicum rotundum), S. 9.
} 
Das Niveau der Vorstellungen der damaligen Ärzte über die Verdauung wird durch die Ansichten des berühmten Boerhaave veranschaulicht, der die Verdauung vom Zusammenwirken vieler nicht in direktem Zusammenhang miteinander stehenden Faktoren abhängig machte: Zerkleinerung und Auflösung von Nahrungsmitteln, Fermentation und Verrottung im Magen u.s.w. Der saure Geschmack von Magensaft wurde zwar festgestellt, dieses Phänomen jedoch nicht auf die sekretorische Aktivität des Magens zurückgeführt, sondern als Ergebnis einer Säurefermentation angesehen.

Die gesamte Geschichte der Untersuchung der Funktionen des Magens von etwa 1650 bis in die frühen 1890er Jahre teilte A.I. Schtscherbakow in drei große Abschnitte ein. Die erste (oder vorbereitende) Periode beginnt in seiner Klassifizierung mit den Werken von Réaumur und Spallanzani und ist gekennzeichnet durch die Tatsache, dass „die Lehre von der Verdauung es erreicht hat, sich vollständig von den Ansichten der alten Autoren mit ihren willkürlichen und oft mystischen Hypothesen zu befreien. In dieser Zeit wurde die eigentliche Rolle der Magensekretion innerhalb des peptischen Prozesses eindeutig bewiesen. “30 Die weitbekannten Experimente von Réaumur zeigten unwiderlegbar, dass nicht die mechanische Arbeit des Magens die Hauptrolle bei der Verdauung spielt, sondern die chemische Wirkung des sezernierten Safts.

Réaumur führte seine Versuche an Vögeln durch, in deren Magen perforierte Metallröhrchen mit Futter eingeführt wurden. Nach einigen Stunden entnahm er die Röhrchen und fand die weich gewordenen Nahrungsstoffe, die sich in eine Breimasse verwandelt hatten, vor. Réaumur erkannte, dass stickstoffhaltige Nahrung durch den Magensaft besonders betroffen war. Weiter stellten die Forscher fest: Wenn das Futter in den Röhrchen durch einen Schwamm ersetzt und dann dessen Inhalt ausgepresst wurde, hatte der ausgepresste Saft einen sauren Geschmack.

Die Bedeutung des Magensaftes für die Verdauung konnte Spallanzani viel überzeugender nachweisen, als er sich folgende Frage stellte: Konnte man eine Vorstellung davon gewinnen, wie der Magensaft die Nahrung im lebendigen Organismus beeinflusst? Dazu benötigte er eine ziemlich große Menge Magensaft. Er nutzte dabei die Methode von Réaumur, also die Einführung von in durchbohrte Röhrchen gelegten trockenen Schwämmen in Vogelmägen; so vermochte er eine genügende Menge Magensaft für weitere Studien zu gewinnen. Innerhalb einiger Tage konnte man 13 Unzen Saft von fünf Krähen erhalten. Zum Erstaunen der Forscher war der Saft wenig flüchtig und nicht entzündlich. Fleischstücke, die für einige Stunden in die aus den Schwämmen gepresste Flüssigkeit gelegt worden waren, wurden bei Körpertemperatur verdaut. Diese Versuchsergebnisse von Spallanzani widerlegten die alten mystischen Ansichten über die Lebenskraft und zeigten, dass der Verdauung im Magen chemische Reaktionen zugrunde liegen. Vorstellungen von der Magenverdauung als Gärungs- bzw. Fäulnisprozess wurden

\footnotetext{
${ }^{30}$ Schtscherbakow, A. I., Über die Bedingungen der Entwicklung des runden Magengeschwürs (Ulcus ventriculi chronicum rotundum), S. 10.
} 
damit ebenfalls widerlegt. Spallanzani wies sogar nach, dass der Magensaft die Fäulnis und die Gärung der Nahrung im Magen nicht etwa anregte, sondern hemmte. Von großer Bedeutung war ferner der Nachweis, dass der Magensaft von den Wänden dieses Organs abgesondert wurde. Spallanzani betrachtete die Magensaftazidität als notwendige Bedingung seiner verdauenden Wirkung.

Der nächste Schritt bei der Erforschung der gastrischen Funktionen und der Bedeutung der Salzsäure für die Verdauung gelang 1824, als Prout zeigte, dass die freie Säure im Magensaft Salzsäure ist. Seine Methode der chemischen Identifizierung des $\mathrm{HCl}$ war kompliziert: Der Magen eines gerade getöteten Kaninchens wurde mit Wasser durchspült. Alle Portionen der Spülflüssigkeit wurden gemischt; diese Mischung wurde in vier Teile getrennt. Bei der folgenden chemischen Analyse stellte sich heraus, dass der Magensaft weder Schwefel- noch Phosphorsäure in nennenswerten Mengen enthielt. Nach Prouts Auffassung ließ sich aus seinen Studien schließen, dass es keine organischen Säuren im Magensaft gibt.

Somit gelangte er zu der unvermeidlichen Schlussfolgerung, dass die Salzsäure die einzige freie Säure des Magens ist. Im Magensaft des Menschen fand er ebenfalls freie Salzsäure. Nach Einschätzung von Schtscherbakow hatte die Studie von Prout eine überragende Bedeutung für die Entwicklung der Lehre vom Magensaft: Das Augenmerk der Wissenschaftler wurde auf den einzig richtigen Weg exakter physiologisch-chemischer Forschungen gelenkt und das überraschende Forschungsergebnis löste ein reges Interesse an entsprechenden Studien aus. Die im Anschluss an Prout durchgeführten Studien bestätigten die Entstehung des Magensaftes in Form einer anorganischen Säure, die unentbehrlich für die Verdauungstätigkeit des Organs war. ${ }^{31}$

Die Erforschung der Sekretionstätigkeit des Magens sowie der chemischen Zusammensetzung des Magensafts wurde dadurch stark erschwert, dass die Wissenschaftler in ihrem methodischen Instrumentarium nicht über die erforderlichen Verfahren zur Magensaftgewinnung verfügten. Für die Untersuchung des Mageninhaltes mussten Versuchstiere getötet werden, nachdem Nahrungsstoffe (oder feste Stoffe zur Anregung der Sekretion) in deren Magen eingefüllt worden waren. So hatten die Experimentatoren keine Möglichkeit, physiologische Versuche anzustellen. Auch sollten bei der Untersuchung Schwämme in den Magen der Tiere eingeführt werden; die Forscher vermochten dabei aber den reinen Magensaft vom Chymus nicht zu trennen. So war es nicht erstaunlich, dass verschiedene Autoren aufgrund ihrer theoretischen Vorstellungen einmal Salzsäure, ein anderes Mal Milchsäure und ein drittes Mal Fettsäuren als einen genuinen und wesentlichen Bestandteil des normalen Magensaftes darstellten.

Eine prinzipiell neue Etappe - Schtscherbakow bezeichnete sie als „laborbezogen“ bei der Erforschung der Wirkungen des Magensaftes und der Bedeutung der Salzsäure für die Verdauung wurde 1842 mit der Erfindung des Gastrostomas durch Wassili

\footnotetext{
${ }^{31}$ Schtscherbakow, A. I., Über die Bedingungen der Entwicklung des runden Magengeschwürs (Ulcus ventriculi chronicum rotundum), S. 13.
} 
Alexandrowitsch Bassow eingeleitet. Als klinisches Vorbild für seine Versuche gilt bekanntlich der von Beaumont beschriebene Fall eines posttraumatischen Gastrostomas (siehe Kap. I). Schtscherbakow bezieht sich auf Literatur, derzufolge der Arzt Helm aus Wien einen ähnlichen Fall bereits 1803 beschrieben hatte. Die Methode der Magenfistelanlegung wurde später von bekannten Physiologen wie Bidder, Schmidt, Claude Bernard, Schiff und anderen ausgebaut und verfeinert; durch diese Weiterentwicklung konnte sie in der täglichen experimentellen und später auch in der klinischen Praxis mühelos angewandt werden.

1852 konnten Bidder und Schmidt reinen Magensaft gewinnen, der beim Necken eines hungrigen Hundes mit Fleisch aus dem Gastrostoma ausgeschieden wurde. Bei der Analyse dieser Flüssigkeit gelang es ihnen zu beweisen, dass freie Salzsäure der wesentliche Bestandteil des reinen Magendrüsensekrets war und der Magensaft keine anderen freien Säuren enthielt. Diese Entdeckung wurde zu einem wichtigen Meilenstein beim Studium der Bedeutung der Salzsäure für die Verdauung sowie für die normale und pathologische Magentätigkeit.

Nach der Meinung von Schtscherbakow zogen die Studien von Bidder und Schmidt eine Bilanz der laborbezogenen Forschungsperiode des Magensaftes und leiteten eine neue Untersuchungsphase der Magenfunktionen sowie der Rolle der Salzsäure ein. Diese Etappe bezeichnete Schtscherbakow als „klinische Periode“ zur Überprüfung der Rolle der Salzsäure bei der Magenverdauung.

Neben der Salzsäure gerieten auch weitere Bestandteile des Magensaftes in den Fokus der Forschung. So zeigte Eberle 1834, dass eine Flüssigkeit mit einem hohen Potenzial zur Verdauung von Eiweißstoffen entstand, wenn man die Magenschleimhaut mit durch $\mathrm{HCl}$ angesäuertem Wasser in Berührung brachte. 1839 entdeckte Schwann im Magensaft das Ferment Pepsin, das Bassmann im gleichen Jahr isolieren konnte.

Mit verbesserten Mikroskopen wurden zur gleichen Zeit die Magendrüsen, die die Salzsäure sezernierten, entdeckt (Sprott 1836; Bischoff 1838; Krause 1839 u.s.w.).

Die Wirkung des Magensaftes auf Nahrungsstoffe und die Untersuchung der Verdauungsprodukte waren ebenfalls Gegenstand mehrerer Studien in diesem Zeitraum (Miahle 1846; Mulder 1858; Brücke 1859 u.s.w.). So gelang es, die wichtigsten Bestandteile des normalen Magensaftes in den 40er und 50er Jahren des 19. Jahrhunderts genau zu bestimmen. Darüber hinaus ,wurden ernsthafte Versuche unternommen, den physiologischen Sekretionsprozess in allen Einzelheiten zu erforschen und die Umwandlungsprodukte verschiedener Stoffe während der Wirkung der Verdauungstätigkeit des Organs zu studieren."32 Schtscherbakow hob dabei hervor, wie wichtig die Verfahren zur Anlage einer Fistel waren, die es den Wissenschaftlern ermöglichten, zu sorgfältigen

\footnotetext{
${ }^{32}$ Schtscherbakow, A. I., Zur Frage nach der Herkunft der freien Salzsäure im Magensaft. Moskau 1890. S. 17.
} 
Beobachtungen $\mathrm{zu}$ gelangen und präzise Versuche anzustellen. ${ }^{33}$ Die beschriebene Periode in der Geschichte der Erforschung der Magenfunktionen zeichnete sich durch das Eindringen der Erkenntnisse zur Magenverdauung in die klinische Praxis aus, wodurch ein gangbarer Weg für Untersuchungen der Erkrankungen des Verdauungssystems gebahnt wurde. Eben aus diesem Grund bezeichnete Schtscherbakow diesen Zeitraum als ,klinische Periode“34. Den Anfang dieses Zeitabschnitts datierte er auf das Jahr 1871, als Leube auf dem Kongress der Naturforscher und Ärzte in Rostock den Vorschlag unterbreitete, eine Magensonde für diagnostische Zwecke einzusetzen. Es ist hervorzuheben, dass Gerhardt noch vor den Versuchen von Leube 1868 auf die Magensonde als diagnostisches Instrument hingewiesen hat.

Einen starken Impuls zur Anwendung dieses Instrumentes in der klinischdiagnostischen Praxis gab Velden mit seinen Studien, mit deren Hilfe er 1879 nachwies, dass die fehlende Salzsäure im Magensaft als wichtiger Baustein bei der Diagnose von Magenkarzinomen gelten konnte. Diese Entdeckung bewies überzeugend die Bedeutung der chemischen Untersuchung des Magensaftes. Bei verschiedenen Erkrankungen wurde nun der Säuregehalt sorgfältig untersucht, und Verfahren zur qualitativen sowie quantitativen Bestimmung der Salzsäure wurden rege erforscht. Aus den Untersuchungen, die innerhalb kurzer Zeit nach Veldens Entdeckung durchgeführt wurden, ergaben sich vor allem - so Schtscherbakow - klinische Methoden zur Magensaftgewinnung (Leube, Riegel, Ewald u.s.w.) und eine ganze Reihe von Verfahren für die sowohl qualitative als auch quantitative Bestimmung von im Magensaft nachzuweisenden Säuren. ${ }^{35}$

Etliche Studien wurden unternommen, um zu beweisen, dass die Salzsäure bei einem Magenkarzinom im Magensaft fehlt. Dabei stieß man ganz zufällig auf einen erhöhten Gehalt dieser Säure bei anderen Magenerkrankungen. Die Analyse der Fälle, die sich durch eine Hypersekretion des Magensaftes und eine erhöhte Azidität des Mageninhaltes auszeichneten, zeigte eine enge Verbindung dieser Zustände mit der Entwicklung des runden Geschwürs. Alles das schien es notwendig zu machen, auf breiter Front Methoden der Magensondenanlage in Kombination mit weiteren chemischen Untersuchungen des über die Sonde ausgeschiedenen Inhalts in die klinische Praxis einzuführen. Die Entwicklung der Magensonde war damals ein guter Grund für Schtscherbakow, den beschriebenen Zeitabschnitt in den Forschungen zur Magenpathologie als „,klinischen“ zu bezeichnen. In dieser ,klinischen“ Periode wurden mit großem Engagement auch Methoden der physiologischen Erforschung der Magenfunktionen

\footnotetext{
${ }^{33}$ Schtscherbakow, A. I., Zur Frage nach der Herkunft der freien Salzsäure im Magensaft. Moskau 1890. S. 17.

${ }^{34}$ Schtscherbakow, A. I., Über die Bedingungen der Entwicklung des runden Magengeschwürs (Ulcus ventriculi chronicum rotundum), S. 18.

${ }^{35}$ Schtscherbakow, A. I., Über die Bedingungen der Entwicklung des runden Magengeschwürs (Ulcus ventriculi chronicum rotundum), S. 19.
} 
entwickelt. Es stellte sich nämlich die Frage: Wie sollen diese oder jene Daten der chemischen Magensaftanalyse bei unterschiedlichen Erkrankungen bewertet werden? Es wurde klar, dass die Rolle der Magensaftsekretion bei unterschiedlichen Formen von Magenerkrankungen nur dann befriedigend geklärt werden konnte, wenn Parameter und Grenzen der physiologischen Norm festgelegt waren.

Viele Forscher interessierten sich für die Magensekretion und ihre Grundlagen, vor allem für den Gehalt der freien Salzsäure im Magensaft nach der Aufnahme verschiedener Nahrungsarten. Dieses Thema wurde in den Studien von Klinikern und Experimentatoren wie Ewald und Boas ausführlich und sorgfältig bearbeitet. Sie nahmen an, dass der Sekretionsprozess nach Nahrungsaufnahme in drei Stadien aufgeteilt werden konnte: Im ersten Stadium beobachtete man nur die Bildung von Milchsäure. Im zweiten Stadium wurden sowohl Milch- als auch Salzsäure sezerniert. Im dritten Stadium war die Sekretion allein der Salzsäure zu beobachten.

Von der Bildung des Magensaftes in den ersten Minuten der Verdauung zeugen auch die Produkte der Eiweißspaltung im Mageninhalt. Schtscherbakow erhob gegen die erwähnten Autoren Einwände und erläuterte, dass die Eiweißspaltung bereits zu Beginn des Verdauungsvorgangs einsetzte. Er stellte fest: „Es sollte angenommen werden, dass die Salzsäure durch die Magenschleimhaut gleich am Anfang zusammen mit Pepsin abgesondert wird; sie mischt sich mit dem Nahrungsbrei, in dem sie auf eine Vielzahl von Stoffen trifft, mit denen sie auf diese oder jene Weise zusammenzuwirken beginnt. "36 Weiter machte er auf die Tatsache aufmerksam, dass Eiweiße den Magensaft besonders stark wirken ließen. Ewald und Boas wiesen gleichzeitig nach, dass der Nahrungsballen eine höhere Azidität als der Mageninhalt hat. Diese Beobachtungen wie auch die Versuche von Bidder und Schmidt zeugten davon, dass der Magensaft mit einer höheren Azidität bereits während der ersten halben Stunde der Verdauung im Magen sezerniert und im Chymus vermischt wird mit den Produkten der Eiweißspaltung, den Peptonen. Die zweite Phase der Verdauung im Magen zeichnete sich nach Angaben von Ewald und Boas durch mehr Peptone sowie eine höhere Chymusazidität aus; zugleich werden die motorische Aktivität des Magens und die Abgabe seines Inhaltes in den Zwölffingerdarm intensiviert.

Aus der Erörterung der Verdauungsphasen im Magen zog Schtscherbakow die Schlussfolgerung, dass die Lehre von den Verdauungsstadien, wie sie vorwiegend von Ewald und Boas entwickelt worden war, als noch nicht vollendet gelten konnte. ${ }^{37}$

Die genannten Autoren hatten in der Tat keine umfassende Vorstellung von den Magenfunktionen, was auf ihre ungenügende theoretische sowie methodische Basis zurückzuführen war. Eine neue Möglichkeit bot sich erst, nachdem Pawlow

\footnotetext{
${ }^{36}$ Schtscherbakow, A. I., Über die Bedingungen der Entwicklung des runden Magengeschwürs (Ulcus ventriculi chronicum rotundum), S. 27.

${ }^{37}$ Schtscherbakow, A. I., Über die Bedingungen der Entwicklung des runden Magengeschwürs (Ulcus ventriculi chronicum rotundum), S. 31.
} 
systematische Verfahren zur Erforschung der Verdauung im Magen und seine Lehre über ihre zwei Phasen, nämlich die psychische und die chemische, entwickelt hatte.

Eine der wichtigen Aufgaben, welche die Forscher zu lösen versuchten, bestand in der Bestimmung des Verhältnisses zwischen Salz- und Milchsäure in verschiedenen Phasen der Magenverdauung. Eine Reihe von Autoren stellte Versuche an und wollte dabei beweisen, dass der Magensaft nicht nur während des Verdauungsvorgangs, sondern auch im nüchternen Zustand produziert wird. Die Ergebnisse dieser Experimente waren widersprüchlich, ihr Sinn aber bestand, so Schtscherbakow, in Folgendem: Wenn Magensaft auch nüchtern gewonnen werden konnte, spielte die individuelle Reizbarkeit des Nervensystems sowohl des Magens selbst als auch des ganzen Organismus die wichtigste Rolle. Einige klinische Fälle zeigten einen allmählichen Übergang zu pathologischen Formen einer „Hypersecretio acida“, bei denen die Magensaftproduktion nicht von zufälligen bzw. externen Faktoren abhing; sie war im leeren Magen unter Einwirkung von der Forschung unzugänglichen Einflüssen zu beobachten, die vom Organismus selbst ausgingen. ${ }^{38}$

Dank der breiten Anwendung der Magensonde bei der Erforschung des Magensaftes entwickelte sich in der Forschung eine neue Richtung, nämlich die Gastroskopie. Schtscherbakow sagte dieser Methode eine große Zukunft voraus. Er war der Auffassung, dass die Gastroskopie eine korrekte anatomische Diagnose ermöglichen werde: „Wenn auch die Qualität der heute zur Untersuchung des Magens per visum vorgeschlagener Instrumente bei weitem nicht zufriedenstellend ist, so haben wir doch allen Anlass, mit bedeutenden Verbesserungen in der Zukunft zu rechnen: Es ist höchst wahrscheinlich, dass wir mit verbesserten gastroskopischen Verfahren irgendwann die sorgfältigste direkte Untersuchung der Magenschleimhaut beim lebenden Menschen werden durchführen können. “39

Diese Vorhersage des russischen Wissenschaftlers ist heute in der klinischen Praxis der Gastroenterologen voll und ganz Wirklichkeit geworden.

Schtscherbakow legte die Geschichte der Magensonde als therapeutische bzw. diagnostische Methode ausführlich dar. Er hob hervor, dass dieses Verfahren von einigen Ärzten bereits Anfang des 19. Jahrhunderts sporadisch angewandt wurde, eine systematische Nutzung jedoch erst nach Kussmauls Studien möglich war. Dieser hatte 1869 vorgeschlagen, den Mageninhalt zwecks Behandlung von Magenerweiterungen auszupumpen. Zuerst legten die Mediziner eine starre Sonde an, die später durch eine weiche ersetzt wurde.

Ewald wurde die Ehre zuteil, die weiche Sonde in die medizinische Praxis einzuführen, als er sie 1875 zum Auspumpen des Mageninhaltes bei einem Kranken mit

\footnotetext{
${ }^{38}$ Schtscherbakow, A. I., Über die Bedingungen der Entwicklung des runden Magengeschwürs (Ulcus ventriculi chronicum rotundum), S. 37-38.

${ }^{39}$ Schtscherbakow, A. I., Über die Bedingungen der Entwicklung des runden Magengeschwürs (Ulcus ventriculi chronicum rotundum), S. 39.
} 
Nitrobenzolvergiftung legte. Das Verdienst von Leube bestand in der systematischen Nutzung der Magensonde zu diagnostischen Zwecken. Seines Erachtens konnte man mit der Sonde zwei Aufgaben gleichzeitig lösen: die Bestimmung 1) der Verdauungszeit im Magen und 2) der Intensität der Magensaftausscheidung in jedem einzelnen Fall.

Für die Bewältigung der formulierten Aufgaben wurden verschiedene Verfahren aufgeboten, mit denen die Sekretion des Magensaftes stimuliert werden konnte. Leube griff zur thermischen Reizmethode der Magenschleimhaut: Er führte $100 \mathrm{ml}$ eiskaltes Wasser für $10 \mathrm{~min}$ in den leeren Magen ein. Dann wurde der Magen mit $300 \mathrm{ml}$ angewärmtem Wasser durchspült. Ein Teil der gewonnenen Flüssigkeit wurde für einen Versuch zur künstlichen Eiweißverdauung genutzt; mit dem anderen Teil wurde die Azidität des Mageninhalts bei Einsatz von Lackmus und Tropeolin geprüft. Diese Methode, so Schtscherbakow, vermittle allerdings nur eine sehr ungenaue Vorstellung über den wirklichen Vorgang der Magensekretion.

Ausgehend von der Überlegung, dass das wichtigste Moment beim Digestionsprozess im Magen die Eiweißspaltung war, veröffentlichten Gluzinski und Jaworski ihr Forschungsverfahren unter der Bezeichnung „Eiweißmethode“. Die Magensekretion wurde durch die Einführung eines physiologischen Reizauslösers, des Hühnereiweißes, angeregt. Ewald unterbreitete seine eigene Version des „Probe-Frühstücks“ für den Fall, dass die Produktion des Magensaftes durch gewöhnliche Mischnahrung erzielt werden sollte. Aus den Werken russischer Autoren, die an diesem Problem gearbeitet hatten, wählte Schtscherbakow Wagners Studien aus der Klinik von W. Manassein, Burshinski, Rapport und N. Shdan-Puschkin aus.

Durch die Analyse der von seinen Vorgängern gesammelten Daten gelangte Schtscherbakow zur Schlussfolgerung, dass die Verdauungszeit ganz wesentlich von den physischen bzw. chemischen Eigenschaften der Nahrung selbst abhing. Er konnte aber die Kriterien zur Beurteilung, wann ein Verdauungsvorgang normal oder pathologisch war, kaum einschätzen. Alle damals bekannten Forschungsmethoden waren nur zur allgemeinen Untersuchung der Magentätigkeit geeignet. So vermittelte z. B. das Verfahren von Leube eine bloße Vorstellung von der Zusammensetzung und den Eigenschaften des reinen Magensaftes. Schtscherbakow zollte Leube dabei hohe Anerkennung und hob hervor, dass das größte Verdienst dieses Wissenschaftlers seines Erachtens eben darin bestand, dass er die Erforschung der Magenverdauung zu diagnostischen Zwecken in den Vordergrund gerückt und somit das Problem aus dem Bereich des physiologischen Labors in die Klinik überführt hatte: „Dadurch war er der Erste, der uns einen absolut richtigen Weg gewiesen hat. Damit meinen wir vor allem seinen Vorschlag, die Qualität des Magensaftes zu erforschen, um eine klare Einsicht in die krankhaften Veränderungen der Sekretionsfunktion des Organs zu erhalten. " 40 Schtscherbakow nahm eine detaillierte Analyse aller vorhandenen Stimulationsverfahren der Magensekretion vor und folgerte,

\footnotetext{
${ }^{40}$ Schtscherbakow, A. I., Über die Bedingungen der Entwicklung des runden Magengeschwürs (Ulcus ventriculi chronicum rotundum), S. 50.
} 
dass sie allein die Frage beantworteten, wann der Magen zur Sekretion der Salzsäure fähig war. Die Frage nach den Einzelheiten des Chemismus der Digestion blieb offen.

$\mathrm{Zu}$ der Zeit, als sich Schtscherbakow mit dem Studium der Ätiologie und Pathogenese der Geschwürkrankheit zu beschäftigen begann, unterstützten bei weitem nicht alle Wissenschaftler die Auffassung, dass der Säuregehalt im Magensaft in einem direkten Zusammenhang mit dieser Erkrankung stand. Er bewertete den Forschungsstand wie folgt: „Die bis heute durchgeführten Forschungen haben ohne Zweifel bloß nachgewiesen, dass Säuren einen sich stark ändernden Bestandteil des Magensekrets besonders unter pathologischen Bedingungen bilden. Die Untersuchungen zur Anomalie der Säuresekretion haben uns bereits sehr wertvolle Daten für Diagnostik und Behandlung geliefert. "41 Eine der wichtigsten Aufgaben der Forscher bestand darin, Methoden für die zuverlässige qualitative bzw. quantitative Bestimmung der Salzsäure im Magensaft zu finden.

Der von Schtscherbakow gewählte Weg zur Feststellung des vorhandenen $\mathrm{HCl}$ im Magensaft führte zu folgendem Ablauf. Das Reagens wurde aus einem Gemisch von zwei Teilen Pyrogallussäure, einem Teil Vanillin und 30 Teilen des mit trockenem $\mathrm{CaSO}_{4}$ dehydrierten Alkohols zubereitet. Das Reagens änderte sich unter Wirkung der organischen Säuren im Magensaft nicht. In der reinen Wasserlösung der Salzsäure löste das Pyrogallol-Vanillin die Reaktion bei einem winzigen $\mathrm{HCl}-$ Gehalt (0,03 bis 0,05 \%) aus. Im Magensaft, der Karzinompatienten entnommen wurde, wies das Reagens in der Regel keine freie Salzsäure nach. Schtscherbakow stellte bestimmte Forderungen an die chemischen Reagenzien; sie mussten es ermöglichen, die Salzsäure im Magensaft zuverlässig festzustellen: „Bei der Einschätzung der relativen Wirkung von qualitativen Reagenzien auf die Magensaftsäuren sollten wir [...] von den notwendigen Eigenschaften einer guten Methode klinisch-chemischer Forschung ausgehen: nämlich Sensitivität, Kennzeichnungsgrad, Spezifität, Reliabilität und endlich leichte Anwendbarkeit am Krankenbett. "“2

Die Bestimmung der vorhandenen Salzsäure im Magensaft mit qualitativen Methoden war nicht die abschließende und auch nicht die allerwichtigste Aufgabe der Kliniker und Pathologen bzw. Experimentatoren. Alle zur Feststellung des $\mathrm{HCl}$ genutzten Reaktionen konnten, so Schtscherbakow, die Frage nach der Menge der Salzsäure, die sich normalerweise und bei verschiedenen Erkrankungen bildet, nicht beantworten. Die Diskussionen unter den Autoren, die die Bedeutung der Salzsäure für die Magenverdauung studierten, waren seiner Meinung nach auf eine ungenügende Aufmerksamkeit den quantitativen Methoden gegenüber zurückzuführen; viele Wissenschaftler erkannten deren Bedeutung schlicht nicht an. Schtscherbakow jedoch bemerkte, dass es bei sehr

\footnotetext{
${ }^{41}$ Schtscherbakow, A. I., Über die Bedingungen der Entwicklung des runden Magengeschwürs (Ulcus ventriculi chronicum rotundum), S. 63.

${ }^{42}$ Schtscherbakow, A. I., Über die Bedingungen der Entwicklung des runden Magengeschwürs (Ulcus ventriculi chronicum rotundum), S. 130.
} 
vielen pathologischen Vorgängen nicht um das völlige Verschwinden des Magensaftes (und damit auch der Salzsäure) ging, sondern nur um quantitative Veränderungen. Das gesammelte Wissen zu diesen quantitativen Veränderungen, d. h. den Schwankungen der Konzentrationen, war äußerst wichtig für die Lösung der Schlüsselfrage nach einer normalen bzw. pathologischen Beschaffenheit der Magensekretion.

Der eventuelle Zusammenhang von Magenerkrankungen mit einer vermehrten Magensaftsekretion wurde bereits 1857 von Trousseau hervorgehoben. Die meisten Autoren neigten jedoch dazu, den erhöhten Salzsäuregehalt im Magensaft für eine zufällige Besonderheit zu halten und nicht als pathogenetischen Faktor zu betrachten. Die im Jahre 1882 veröffentlichten Beobachtungen des Arztes Reichmann aus Warschau waren von großer Bedeutung, um die Rolle der Salzsäure verstehen zu können; er beschrieb eine anfallsartiges Magenleiden, dem eine verstärkte Magensaftsekretion zugrunde lag. Der gleiche Autor wies 1884 in seinem Aufsatz „Über saure Dyspepsie“ auf die Häufigkeit dieses Leidens hin, bei dem saures Aufstoßen, Sodbrennen und andere Symptome durch eine $\mathrm{zu}$ frühe und $\mathrm{zu}$ reichliche Magensaftproduktion verursacht wurden. Die Azidität im Intervall zwischen drei und fünf Stunden nach der Nahrungsaufnahme erreichte bei seinen Patienten Werte, welche die normalen Zahlen wesentlich übertrafen. Das medizinische Denken war damals aber noch nicht soweit, um die Bedeutung einer verstärkten Magensaftsekretion für die Pathogenese von Magenerkrankungen zu erfassen. Manche Ärzte empfahlen sogar, Salzsäure als Heilmittel sowohl gegen Magenkrebs als auch gegen Geschwüre anzuwenden. Andere Mediziner verschrieben verschiedene alkalische Substanzen zur Ulkustherapie. Schtscherbakow urteilte, dass ,die Anpreisung von zwei absolut gegensätzlichen Mitteln bei dem wahrscheinlich gleichen Leiden wohl äußerst charakteristisch dafür war, dass es selbst in der jüngsten Vergangenheit noch keine genauen und klaren Befunde bei der Behandlung von Magenkrankheiten gab. “43

1889 veröffentlichte Noorden die Versuchsergebnisse, bei denen ein Zusammenhang im Verhältnis zwischen der Magensaftazidität und dem neutralisierenden Effekt des Blutes festgestellt wurde. Dieses Resultat sah Schtscherbakow als wichtigen Fortschritt beim Studium der Ätiopathogenese der Ulkuskrankheit an. Aus allen denkbaren Blickwinkeln analysierte er die Arbeiten jener Autoren, die Forschungen in dieser Richtung vorgenommen hatten. Alle von Noorden und anderen Wissenschaftlern durchgeführten Untersuchungen hatten jedoch einen großen Nachteil: Während der Studien änderte sich der Gegenstand selbst, nämlich die alkalische Blutreaktion, sodass sich die ermittelten Ergebnisse nicht eindeutig interpretieren ließen. Alle Verfahren zur Messung der alkalischen Blutreaktion, ,wenn man auch verschiedene kleine Mängel dabei beiseite

\footnotetext{
${ }^{43}$ Schtscherbakow, A. I., Über die Bedingungen der Entwicklung des runden Magengeschwürs (Ulcus ventriculi chronicum rotundum), S. 237.
} 
lassen würde [...], teilten eine wichtige Ungenauigkeit; sie zeigte sich eben darin, dass man überall mit dem Blut eines sterbenden Lebewesens, dessen Alkaligehalt sich schon während des Analysevorgangs verändern konnte, zurechtkommen musste. “44

Die wichtigste Aufgabe, die sich Schtscherbakow stellte, bestand somit aus der Suche nach einem Verfahren, um die Variabilität des Objektes während des Untersuchungsprozesses zu minimieren. Eine der richtungsweisenden Erkenntnisse seiner Forschungen zur Ätiologie bzw. Pathogenese des runden Geschwürs war der Nachweis der Fähigkeit des Blutes, die Salzsäure des Magensaftes zu neutralisieren.

Keiner der Forscher, die vor Schtscherbakow gewirkt hatten, vermochte ein pathogenetisches Modell der Geschwürkrankheit zu entwerfen, das eine umfassende Wechselwirkung von Kardinalfaktoren berücksichtigte - Kardinalfaktoren, welche die Entstehung bzw. den Verlauf dieser Erkrankung bestimmten. Die ausführliche Übersicht und die Analyse der historischen Grundströmungen der Forschung im 19. Jahrhundert war für Schtscherbakow gleichzeitig Vorbereitung auf und Ausgangspunkt für die darauf folgende experimentelle Behandlung des Problems - und zwar auf der Basis eines umfassenden theoretischen Modells dieser Pathologie.

\section{Zur Theorie über die Entstehung und Entwicklung des Magengeschwürs von A. I. Schtscherbakow}

Wie soeben dargestellt, hatte kein Forscher vor Schtscherbakow eine ganzheitliche Theorie zu Ursache und Entwicklung des Geschwürs geschaffen, die allen der Wissenschaft bekannten Tatsachen Rechnung getragen und auf genau festgelegten und beim Experiment nachvollziehbaren Angaben beruht hätte. Cruveilhier, Rokitansky und Virchow hatten ihre Theorien auf der Grundlage der pathoanatomischen Befunde errichtet; sie hatten das Wesen der krankhaften Prozesse nach dem postmortalen Bild des ausgebildeten Geschwürs beurteilt. Deshalb trugen ihre Gedankengebäude einen stark hypothetischen Charakter hinsichtlich der Erklärung der Pathogenese und der Ätiologie dieser Erkrankung. Zudem erläuterten sie nur die Entstehung der lokalen Schädigung der Magenwand - was das Wesen des Geschwürs als spezifische nosologische Ausprägung nicht erkennen ließ. „In der Lehre über die Ätiologie des runden Magengeschwürs“, formulierte Schtscherbakow, ,ist es notwendig, beide Fragen, die nach der Entstehung des Strukturschadens sowie die nach der weiteren Entwicklung und dem progredienten Verlauf zu trennen. Die Theorien, die diesem Umstand keine Rechnung tragen, sind gar nicht in der Lage, Licht in den Bereich der Lehre vom runden Magengeschwür zu bringen." 45

\footnotetext{
${ }^{44}$ Schtscherbakow, A. I., Über die Bedingungen der Entwicklung des runden Magengeschwürs (Ulcus ventriculi chronicum rotundum), S. 365.

${ }^{45}$ Schtscherbakow, A. I., Über die Bedingungen der Entwicklung des runden Magengeschwürs (Ulcus ventriculi chronicum rotundum), S. 458.
} 
Virchows Gedanken, dass neben der Ulzeration an der Ätiologie und Pathogenese des Geschwürs auch andere Faktoren mitwirken, hatten den Charakter rein theoretischer Annahmen, die weder durch klinische Beobachtungen noch durch experimentelle Erkenntnisse bestätigt wurden. Die Untersuchungen des Magensafts und der Magensaftazidität bei unterschiedlichen Magenerkrankungen erschienen erst 20 bis 30 Jahre nach den Arbeiten Virchows, als die Untersuchung mittels der Magensonde in die diagnostische Praxis eingeführt wurde.

Das von Cohnheim entwickelte Modell war ein markanter Schritt vorwärts bei der Untersuchung der Ursachen des Magengeschwürs. Die Gefäßfaktoren, die zur Ulzeration führen und die die Forscher schon vor Cohnheim diskutiert hatten, wurden von ihm experimentell erzeugt und reproduziert. Zwar hat auch Cohnheim auf die Rolle der Magensaftazidität verwiesen, aber er hat diesen Faktor nicht experimentell untersucht und ihn nicht in die logische Struktur seines Modells eingeführt. ${ }^{46}$

Das war die erste Gruppe von Untersuchungen, deren Ergebnisse Schtscherbakow bei der Schaffung seines Konzeptes nutzte.

Das Hauptmoment der historischen Entwicklung betreffs der Forschungen zur Ätiopathogenese des Geschwürs stellte die Entdeckung einer erhöhten Magensaftazidität (Riegel, Hirsch, Jaworski u. a.) dar. Aber diese Entdeckung allein genügte ebenfalls nicht, um ein multifaktorielles Modell zu konstruieren.

Man benötigte eine Konzeption, welche Ursache und Entstehung des Geschwürs als einen ganzheitlichen Komplex unter Berücksichtigung aller bekannten Faktoren auffasst. Schtscherbakow stellte fest, dass „,das Vorhandensein einer erhöhten Menge von Salzsäure im Magen [...] schon allein genügt, um nicht nur die Störung der Unversehrtheit [der Schleimhaut] zu erzeugen, sondern auch, um diese Störung fortschreiten zu lassen und letztendlich [die Magenwand] zur Perforation zu bringen. “47

Zur gleichen Zeit verstand er, dass auch dieser Faktor nicht die einzige Ursache für die Entstehung des Geschwürs sein kann. A.I. Schtscherbakow bemerkte zu Beginn seiner Arbeit an diesem komplexen Problem, dass Professor A.I. Voigt vorgeschlagen habe, „das Thema zu erweitern und in die Versuchsreihe einen anderen wichtigen Faktor einzuführen, der eine fortschreitende Ulzeration auslösen könne - nämlich die Schwächung der Gegenreaktion des Gewebes."48 Aufgrund dieses Vorschlags und der bestehenden klinischen und experimentellen Daten entwickelte Schtscherbakow eine Forschungsstrategie, in deren Verlauf sich die Ausgangshypothese in eine gut aufgebaute und experimentell bestätigte Theorie verwandeln sollte. Das wesentliche Problem, das am Ende aller Untersuchungen gelöst werden sollte, formulierte er wie folgt: „Warum

\footnotetext{
${ }^{46}$ Cohnheim, J. F., Untersuchungen ueber die embolischen Processe. Berlin, 1872.

${ }^{47}$ Schtscherbakow, A. I., Über die Bedingungen der Entwicklung des runden Magengeschwürs (Ulcus ventriculi chronicum rotundum), S. 335.

${ }^{48}$ Schtscherbakow, A. I., Über die Bedingungen der Entwicklung des runden Magengeschwürs (Ulcus ventriculi chronicum rotundum), S. 4-5.
} 
neigt - im Unterschied zu einfachen Störungen der Intaktheit des Magens mit einer schnellen Heilung - das Ulcus rotundum ventriculi zu einem fortschreitenden Verlauf, der gelegentlich sogar zu einer Perforation fuhrt?“49

Bei der Wahl des experimentellen Modells, mit dessen Hilfe man die Rolle und das Zusammenwirken aller zu diesem Zeitpunkt bekannten Faktoren untersuchen konnte, legte Schtscherbakow Cohnheims Untersuchungen zugrunde: „Cohnheims Versuche“, so Schtscherbakow, ,stellen einen Ausgangspunkt für die Weiterentwicklung der gegenwärtigen Lehre vom Ulcus ventriculi dar. Es ist Cohnheim gelungen, die Gefäßäste der Mucosa und Submuscosa völlig zu verschließen, indem er in eine der Arteriae gastricae breves eine Emulsion von chromsaurem Blei eingespritzt hat. “50

Ein weiteres Verdienst dieses Wissenschaftlers sah Schtscherbakow darin, dass er „mit außerordentlicher Genauigkeit die Entstehung der Ulzeration und deren fortschreitenden Verlauf, welcher die kennzeichnende Besonderheit des Leidens darstellt, trennt. So sehr die erste Frage schon damals mehr oder weniger gelöst erschien, so sehr musste sich Cohnheim in Bezug auf die zweite Frage auf Vermutungen und Annahmen beschränken. “51

Ausgehend von den ihm zur Verfügung stehenden praktischen Erkenntnissen und theoretischen Annahmen schuf Schtscherbakow ein experimentelles Modell. In dieses Modell integrierte er - in einer bestimmten Reihenfolge und in bestimmten Zeitabschnitten - sowohl die Faktoren, die die primäre Ulzeration der Schleimhaut hervorrufen, als auch jene, die zur Verwandlung dieser Ulzeration in ein echtes Ulcus ventriculi rotundum beitragen.

Um lokale Störungen der normalen Kontinuität der Magenwand zu erzeugen, nutzte Schtscherbakow Cohnheims Verfahren, das aus einer Verstopfung der kleinen Blutgefäße des Magens bestand, und zwar mithilfe einer eingespritzten Emulsion aus chromsaurem Blei. Schtscherbakow entwickelte seine eigene Methodik für die Emulsionsherstellung. Er nutzte ein Präparat, dessen Kristalle im Mikroskop eine moosartige Konfiguration zeigten und aus Nadeln mit einer Größe von 0,0045 bis 0,0060 mm bestanden. Das Einspritzen der Emulsion vollzog sich folgendermaßen: Nach Eröffnung des Abdomens und Darstellung des Magens wurde an der großen Magenkurvatur die Eintrittsstelle der kleineren Gefäßäste aufgesucht, die das Organ mit Blut versorgen. Eine dieser Adern wurde von den anderen aus einem Bündel von Venen und Nerven isoliert. Des Weiteren wurden in die abpräparierte Arterie $0,2 \mathrm{~cm}^{3} 5 \%$ ige Emulsion eingeführt, die $0,02 \mathrm{~g} \mathrm{PbCrO}_{4}$ enthielt. Nach der Verabreichung wurde oberhalb der Aderkanüle eine

\footnotetext{
${ }^{49}$ Schtscherbakow, A. I., Über die Bedingungen der Entwicklung des runden Magengeschwürs (Ulcus ventriculi chronicum rotundum), S. 5.

${ }^{50}$ Schtscherbakow A. I., Über die Bedingungen der Entwicklung des runden Magengeschwürs (Ulcus ventriculi chronicum rotundum), S. 289.

${ }^{51}$ Schtscherbakow A. I., Über die Bedingungen der Entwicklung des runden Magengeschwürs (Ulcus ventriculi chronicum rotundum), S. 290.
} 
Ligatur angelegt. Bei der auf diese Weise erreichten Konzentration der Emulsion von chromsaurem Blei entstand eine klar ausgeprägte Störung der Kontinuität der Magenwand, die aber nicht zu einer Perforation führte.

Es zeigte sich, dass die innerhalb der ersten 24 Stunden entstandene Ulzeration alle typischen Merkmale eines frischen Magengeschwürs aufwies: schroff ausgeschnittene Ränder, stufenartiger Aufbau, unversehrter Boden und eine konusartige Form der Läsion bei vollständigem Fehlen einer Entzündungsreaktion im Umfeld. 72 Stunden nach dem Einspritzen von $\mathrm{PbCrO}_{4}$ wurde im Magen des getöteten Hundes eine ziemlich starke lokale Gewebereaktion im Umkreis der Geschwürbildung festgestellt. Diese Reaktion bedeutete den Beginn der Verheilung des akuten Ulkus. Fünf Tage nach der Verabreichung der Emulsion wurde eindeutig der Beginn der Geschwürheilung und einer Verminderung des Entzündungsprozesses festgestellt. Zwei Wochen später war die Oberfläche des ursprünglichen Ulkus mit einer ziemlich glatten Narbe bedeckt. Wenn das Tier für zwei Wochen nach dem Zeitpunkt des Eingriffs am Leben geblieben war, so war in der Regel auch die Narbe am geheilten Geschwür fast mit Schleimhaut bedeckt. Die Schädigung der Magenwand bei gesunden Tieren verheilte vollständig und ausnahmslos nach einem Verlauf von drei Wochen. Die Ulzerationen, die unter der Einwirkung des eingespritzten $\mathrm{PbCrO}_{4}$ entstanden waren, glichen jenen Veränderungen, die beim Magengeschwür des Menschen festgestellt werden; sie waren aber nicht damit identisch. Denn ein experimentelles Geschwür entwickelte sich nicht fortschreitend; es heilte sehr rasch, ohne Spuren zu hinterlassen. Diese Experimente zeigten klar, dass sich primäre Ulzerationen an und für sich ohne Intervention anderer pathogener Faktoren nicht in ein Ulcus ventriculi rotundum verwandeln.

Des Weiteren stellte sich die Aufgabe, den Einfluss von Magensaft mit einer erhöhten Azidität auf die Verheilung der durch eingespritztes $\mathrm{PbCrO}_{4}$ erzeugten Läsion festzustellen. Diese Versuche wurden wie folgt durchgeführt: Gleichzeitig mit der Injektion von chromsaurem Blei in eine der Aa. gastricae breves (zwecks der Erzeugung eines Geschwürs) wurde der Magen in die Wunde der Bauchwand eingenäht, wie dies in ähnlicher Weise für die Anlage einer Fistel vorgenommen wird. Innerhalb der ersten Woche nach dem Einspritzen von $\mathrm{PbCrO}_{4}$ wurde der Magen eröffnet und ein Fistelrohr eingesetzt, über das Salzsäure verabreicht wurde. Zu einem bestimmten Zeitpunkt nach der Injektion des chromsauren Bleis wurde das Ulkus untersucht und es wurde - ebenfalls zu definierten Zeitpunkten - dreimal pro Tag mithilfe des Fistelrohrs eine 4 \%ige Salzsäurelösung in den Magen verabreicht. Das Infusionsverfahren gestaltete sich wie folgt: Am ersten Tag dreimal je $200 \mathrm{~cm}^{3} \mathrm{HCl}$; am zweiten Tag $1000 \mathrm{~cm}^{3} \mathrm{HCl}$. Am Morgen des dritten Tags traten beim Öffnen des Fistelrohrs etwa $200 \mathrm{~cm}^{3}$ einer stark sauren Flüssigkeit aus, die Kaffeesatz ähnelte. Im Laufe des dritten, vierten und fünften Tages sowie der nachfolgenden Tage wurde die Zuführung von $\mathrm{HCl}$ wiederholt. Am neunten Tag starb der Hund. Bei der Autopsie zeigte sich, dass sich an der Magenwand nahe der Einspritzungsstelle ein Geschwür entwickelt hatte, von länglich-ellipsenartiger Form, abgestuft und mit unversehrtem Boden. An einer randständigen Stelle des Ulkus war ein Gerinnsel nachweisbar, welches das Gefäß großkalibrig verstopfte; daneben sah man die 
Stelle der Ulkusperforation in die Bauchhöhle ,,in 10-Kopeken-Größe“ (Durchmesser ca. $1,7 \mathrm{~mm})$.

Wenn die Infusion der Säure in den Magen nicht sofort, sondern zwei Wochen nach der Injektion von $\mathrm{PbCrO}_{4}$ vorgenommen wurde und solche Infusionen drei Wochen lang fortgesetzt wurden, stellte man bei der Öffnung des Organs folgenden Befund fest: Die Schleimhaut des Magens war nur den Folgen einer katarrhalischen Entzündung ausgesetzt, das Geschwür schien jedoch vollständig verheilt zu sein.

Der Vergleich der Ergebnisse beider Versuchsserien zeigte, dass die Erhöhung des Säuregehalts ,zu der fortschreitenden Entwicklung und dem anhaltenden Verlauf der sich auf diese oder jene Weise herausbildenden Ulzeration beiträgt. "52

Bei diesen Experimenten wurde eine Situation simuliert, die die Bedeutung des Zusammenwirkens von zwei Schlüsselaspekten der Pathogenese einer Ulkuskrankheit erkennen lässt: die primäre Ulzeration der Magenwand und die erhöhte Magensaftazidität. Auf diese Weise wurde die unmittelbare Ursache (Causa proxima) der Entwicklung eines Ulcus ventriculi festgestellt.

Bei der Zusammenfassung seiner Versuchsergebnisse kam Schtscherbakow zu einer allgemeinen Schlussfolgerung: „Die Entstehung des Geschwürs ist in den meisten Fällen mit der Störung der Blutversorgung in einem bestimmten Bereich der Magenwand verbunden. [...] Die Störung der Unversehrtheit der Magenwand bedeutet bei weitem noch kein Geschwür, sie verheilt [...] in Form einer glatten Narbe innerhalb sehr kurzer Zeit. Damit sich eine solche Verletzung zu einem Ulcus ventriculi entwickelt, ist die Einwirkung definierter Faktoren erforderlich; als solche sind auf Grund bestehender klinischer Erkenntnisse und einiger Versuche die Erhöhung der Magensaftazidität und die der Resistenzschwäche des Gewebes auszumachen. “53

Kurz danach stand der Wissenschaftler vor einer noch komplizierteren Aufgabe: nämlich der Auswertung der klinischen Fälle der Ulkuskrankheit - ausgehend von der experimentellen, oben beschriebenen Konzeption.

Eine verminderte Resistenz der Magenwand wird im Experiment vom Wissenschaftler künstlich modelliert. Unter natürlichen Bedingungen, so Schtscherbakow, konnten anomale Bedingungen des Stoffwechsels zu ursächlichen Einflüssen werden: „Die Faktoren, die den fortschreitenden Prozess der Ulzeration beeinflussen können, [...] sind auch nach Klärung der Bedeutung von sekretorischen Anomalien bei weitem nicht hinreichend erfasst: Die Entwicklung des Magengeschwürs, sein chronischer Verlauf und Schwierigkeiten bei seiner Heilung können nicht nur durch sozusagen äußere Einwirkung, d. h. durch die ungewöhnlichen Reizung durch zu sauren Magensaft, bedingt sein, sondern auch durch anomale trophische Bedingungen der Organwand selbst; diese

\footnotetext{
${ }^{52}$ Schtscherbakow, A. I., Über die Bedingungen der Entwicklung des runden Magengeschwürs (Ulcus ventriculi chronicum rotundum), S. 335.

${ }^{53}$ Schtscherbakow, A. I., Über die Bedingungen der Entwicklung des runden Magengeschwürs (Ulcus ventriculi chronicum rotundum), S. 317.
} 
führen zur Schwächung ihrer vitalen Funktionen, zur Verminderung der Resistenz gegen schädliche Einwirkungen. Die Möglichkeit der Entwicklung derartiger Ulzerationen entspricht der Pathologie im Bereich anderer Organe des menschlichen Körpers. "54

Die Forschungen zu dieser Frage begannen erst gegen Ende der 1880er Jahre. Zu dieser Zeit lagen nur einzelne Beobachtungen und wenige experimentell gesicherte Erkenntnisse vor, die von einer engen Verbindung des Magengeschwürs mit allgemeinen Ernährungsstörungen des Organismus ausgingen. Dazu zählte Schtscherbakow QuinckeDaettwylers und Silbermans Versuche sowie den von mehreren Klinikern festgestellten Zusammenhang zwischen der Magenulkuskrankheit und der Chlorose sowie der Anämie.

Zur Lösung der Frage nach einem Zusammenhang zwischen einem Ulcus ventriculi und einer Störung der Gewebetrophik der Magenwand führte Schtscherbakow Experimente durch, welche die Bedeutung der Veränderungen der Blutversorgung für die Entstehung des Geschwürs ans Tageslicht brachten.

Die experimentelle Forschung auch zu dieser Frage gestaltete sich anspruchsvoll, denn es war äußerst schwierig, ,die Gewebe eines unmittelbaren Manipulationen schwer zugänglichen Organs, wie es der Magen ist, den Bedingungen anomaler Ernährung auszusetzen, ohne dabei gleichzeitig die Unversehrtheit des Organismus gröblichst zu verletzen. Wir können auf bestimmte Bereiche des Nervensystems, die eine Rolle im Ernährungsprozess der Magenwand spielen, nicht unmittelbar einwirken, denn beim jetzigen Kenntnisstand über trophische Prozesse im Allgemeinen und über die Ernährung der Magenwand im Besonderen scheint ein solcher Weg höchst unsicher zu sein. “55

Unter Berücksichtigung der komplizierten Zusammensetzung des Blutes erschien es notwendig, ein Element auszumachen, das bei der Untersuchung der Verbindung zwischen einem pathologischen Zustand des hämatologischen Systems und dem Entstehungsprozess eines Magengeschwürs hilfreich sein konnte. Viele Forscher waren der Meinung, dass diese Wechselbeziehung mithilfe der Untersuchung der im Blut enthaltenen basischen Stoffe, die als Neutralisatoren der Säuren des Magensafts wirkten, entschlüsselt werden kann. Schtscherbakows Forschungen zielten daher unmittelbar darauf ab, die Verbindung zwischen den Veränderungen der alkalischen Blutreaktion und der Entwicklung eines Magenulkus festzustellen. Dazu führte er ein Experiment durch: Zuerst wurde nach Cohnheims Verfahren eine akute Ulzeration der Magenwand erzeugt. Die Feststellung der alkalischen Blutreaktion erfolgte mithilfe des Titrierungsverfahrens, das von Zuntz, Lassar und anderen entwickelt worden war und vom Anwender leicht modifiziert wurde, um einige Mängel zu beseitigen. Schtscherbakow erkannte, dass bei den damals bekannten Untersuchungsmethoden keine Rede von der Festlegung absoluter Größen bei der alkalischen Blutreaktion sein konnte. Diese Größen waren immer

\footnotetext{
${ }^{54}$ Schtscherbakow, A. I., Über die Bedingungen der Entwicklung des runden Magengeschwürs (Ulcus ventriculi chronicum rotundum), S. 342.

${ }^{55}$ Schtscherbakow, A. I., Über die Bedingungen der Entwicklung des runden Magengeschwürs (Ulcus ventriculi chronicum rotundum), S. 335.
} 
eine Funktion des bei der Analyse benutzten Indikators. Im Hinblick auf die Ziele des Schtscherbakow'schen Experiments waren die Verfahren von Bedeutung, welche die Grenzen der alkalischen Blutreaktion festlegten, die einer Konzentration von 164,4 bis $178,2 \mathrm{mg} \mathrm{Na} \mathrm{O}_{2} \mathrm{O}$ pro $100 \mathrm{ml}$ Blut entsprachen. Bei Verabreichung einer Säure verminderte sich die Alkaligehalt im Blut auf 109,1 mg.

Die Blutuntersuchung bei Patientinnen und Patienten mit verschiedenen Erkrankungen zeigte, dass die genannte Komponente innerhalb eines breiten Spektrums schwankt. Zum Beispiel ermittelten May und Tassinavi bei Diabeteskranken einen Wert von $265 \mathrm{mg} \mathrm{Na} \mathrm{N}_{2}$ je $100 \mathrm{~cm}^{3}$ Blut (Norm: $400 \mathrm{mg} / 100 \mathrm{~cm}^{3}$ ). Eine Verminderung der Alkalireaktion wurde bei Fällen von Infektionsnephritis, Sepsis und onkologischen Krankheiten festgestellt. Man konnte also schlussfolgern, dass bei verschiedenen Erkrankungen die alkalische Blutreaktion deutlichen Schwankungen unterliegt.

Aufgrund der Analyse bekannter Untersuchungsmethoden der alkalischen Substanzen im Blut kam Schtscherbakow zur Schlussfolgerung, dass alle diese Verfahren ,eine ihnen wesenseigene und sehr relevante Ungenauigkeit in sich bergen; sie besteht gerade darin, dass man es überall mit einem sterbenden Blut zu tun haben muss, dessen Alkaligehalt sich schon während des eigentlichen Verlaufs der Analyse ändern kann. " 56 Um verlässlichere und vergleichbare Ergebnisse zu erzielen, brachte er die Idee auf, ein mehr oder weniger „konstantes“ Untersuchungsobjekt zu nutzen und dessen Veränderlichkeit während des eigentlichen Experiments so zu umgehen. Dies war seiner Meinung nach nur durch ein schnelles „Absterben“ (ein Ausdruck von Schtscherbakow) des Blutes mithilfe einer Reaktion desselben mit einer konzentrierten Lösung von indifferentem Salz $\mathrm{zu}$ erreichen. Um die Richtigkeit eines solchen Vorgehens zu rechtfertigen, berief er ich auf die Praxis der Histologen, die die Natur eines lebenden Gewebes untersuchten, indem sie es in Osmiumsäure eintauchten; diese Säure tötete augenblicklich seine Bestandteile $a b$ und fixierte sie verlässlich.

Unter Berücksichtigung aller in der Fachliteratur vorhandenen Kenntnisse und auf Basis des oben beschriebenen Prinzips eines schnellen „Blut-Absterben-Lassens“ schuf Schtscherbakow seine eigene Methode zur Erkennung von basischen Substanzen im Blut; dabei kam eine Reaktion mithilfe von schwefelsaurem Magnesium $\left(\mathrm{MgSO}_{4}\right)$ zur Anwendung.

Die Vorteile seines Verfahrens sah er in der Konstanz des Untersuchungsobjektes im Verlauf der eigentlichen Untersuchung: Die Flüssigkeit, in der die Alkalimenge bestimmt wurde, beeinflusste die Farbe des Lackmuspapiers nicht; die Menge des untersuchten Bluts wurde genau festgelegt; die Flüssigkeit enthielt Eiweißstoffe und Blutfarbstoffe nur in Form von Spuren, die ihre Reaktion nicht veränderten. Es ist offensichtlich, dass die so erzielte Konstanz des Untersuchungsobjekts es möglich machte, Fehler zu vermeiden, die anderen Methoden notwendigerweise innewohnten. Unter Verwendung

\footnotetext{
${ }^{56}$ Schtscherbakow, A. I., Über die Bedingungen der Entwicklung des runden Magengeschwürs (Ulcus ventriculi chronicum rotundum), S. 365.
} 
dieses Verfahrens wurden Durchschnittsblutangaben bei einem gesunden Hund wie folgt ermittelt: 104,3 \% Hämoglobin, 7.370.000 Erythrozyten, alkalische Serumreaktion $73,4 \mathrm{mg} \mathrm{Na} 2 \mathrm{O}$.

Schtscherbakow untersuchte auch die Bedeutung einer künstlich erzeugten Anämie und einer Vergiftung der Tiere durch Pyrogallussäure und Anilin. Dabei wählte der Autor als Indiz die Veränderung der alkalischen Blutreaktion, deren neutralisierende Wirkung gegenüber dem $\mathrm{HCl}$ des Magensafts für ihn am offensichtlichsten war.

Das Ziel aller dieser Versuche war die Simulation sich langsam entwickelnder Anomalien des hämatopoetischen Systems, die einen ungünstigen Verlauf der Magengeschwüre beim Menschen hervorrufen. Eine Anämie bei Tieren mithilfe des Aderlasses zu verursachen, erwies sich praktisch als unmöglich. Deswegen verwendete Schtscherbakow Giftstoffe, deren Anwendung zu einer gleichmäßigen langsamen Zerstörung der Blutzellen führte. Die Folgen waren einer essenziellen Anämie zum Verwechseln ähnlich.

Bei den Versuchen wurde das Anilin einem Versuchshund subkutan als Gemisch mit der gleichen Menge reinen Mandelöls verabreicht. Die Dosis wurde für jeden Einzelfall passend ausgewählt und betrug 0,05 bis 0,1 mg Anilinöl je kg Körpergewicht. Eine solche Dosis beeinflusste fast nie den Allgemeinzustand der Tiere. Vor Beginn des Versuchs wurden das Körpergewicht des Hundes sowie die Hämoglobinmenge und die Erythrozytenzahl pro $\mathrm{mm}^{3}$ Blut bestimmt.

Die Experimente wurden wie folgt durchgeführt: Nachdem das Gewicht des Tieres, die Hämoglobin- und Erythrozytenmenge und die alkalische Blutreaktion bestimmt worden waren, wurde das Anilin in der Menge 0,05 bis 0,1 mg je Kilogramm Körpergewichts eingespritzt. Die Injektion erfolgte in bestimmten Zeitabständen, oft nach jeweils einer Woche. Als die Ergebnisse, die bei der Blutuntersuchung gewonnen worden waren, zeigten, dass eindeutige Veränderungen in seiner Zusammensetzung eintraten, wurde die Blutalkaleszenz bestimmt und $\mathrm{PbCrO}_{4}$ in eine der Arteriae gastricae breves eingespritzt. Zwei bis drei Wochen später wurden erneut das Gewicht des Tieres sowie die Hämoglobin- und Erythrozytenmenge bestimmt; danach begann man mit einer neuen Serie der Anilininjektionen. Zum Abschluss des Experiments wurden wiederum das Gewicht des Tieres sowie die Hämoglobin- und Erythrozytenwerte, auch der Alkaligehalt des Blutes sorgfältig bestimmt; dann ließ man das Tier sterben.

Schtscherbakows Versuchsfolge zeigte, dass bei der Anilinverabreichung die Abheilung des akuten experimentellen Geschwürs nicht erfolgte, was auf die unmittelbare Resistenzminderung des Magengewebes bei der Anilinvergiftung hinwies. Er folgerte, dass die „Anämie, die durch chronische Anilinvergiftung verursacht wurde, Bedingungen im Organismus des Tieres schafft, die zur Entwicklung des Geschwürs beitragen und zur gleichen Zeit die Heilung der einmal herausgebildeten Ulzeration 
stören. " ${ }^{57}$ Aber diese Versuche brachten keine eindeutige Antwort auf die Frage nach der Rolle, welche die Veränderungen der alkalischen Blutreaktion bei der Entwicklung des Geschwürs spielten.

Experimente unter Einsatz von Pyrogallussäure ließen auch erkennen, dass die Ulzeration der Magenwand bei den so vergifteten Hunden nicht innerhalb einer kurzen Frist verheilt. Sogar 30 (und mehr) Tage nach der Geschwürbildung verschwand die Läsion der Schleimhaut nicht. Aber die schwächende Wirkung von Pyrogallol auf die Resistenz der Magenwand gegen schädliche externe Agentien war weniger ausgeprägt als bei der Anilinvergiftung.

Aus allen durchgeführten Versuchen zog Schtscherbakow den Schluss, dass die verminderte Magenwandresistenz nicht allein auf die gestörte Neutralisationsfähigkeit des Blutes im Hinblick auf seine alkalisierenden Valenzen zurückgeführt werden kann. Unmittelbare Veränderungen, vor allem bei den Hämoglobin- und Erythrozytenkonzentrationen, waren sowohl bei der Anilin- als auch bei der Pyrogallolintoxikation zu verzeichnen.

Bei einer Verbindung von Aderlass und Pyrogallolvergiftung heilte bei den Versuchstieren die Magenschädigung langsam, d. h. die Resistenz der Magenwand war geringer. Dabei gingen die Schwankungen der alkalischen Blutreaktion nicht über die Grenzen von Beobachtungsfehlern hinaus. „Der Verminderung des Basengehalts des Blutes, die manchmal durch einen Aderlass hervorgerufen wird, kann man“, so Schtscherbakow, „keine vorrangige Bedeutung dafür beimessen, dass die Heilung der Läsionen nur langsam voranschreitet. “58

Um die „finale Ursache“ für die Geschwürbildung festzustellen, startete er eine andere Versuchsserie mit dem Ziel, den Einfluss der Störung der Blutzusammensetzung auf die Beschaffenheit und die Eigenschaften des Magensafts zu studieren.

Diese Versuche wurden in folgender Weise durchgeführt: In der ersten Phase wurde dem Tier eine Magenfistel angelegt. Nach diesem Eingriff wurde es längere Zeit bis zu dem Punkt beobachtet, an dem es ein konstantes Gewicht hielt. In der zweiten Phase wurden, wie bei den vorausgegangenen Versuchen, regelmäßig das Gewicht des Tieres, Hämoglobinwert, Erythrozytenzahl und pH-Wert des Blutes gemessen. Die Magensaftanalyse wurde in verschiedenen Stadien der Verdauung vorgenommen. Nachdem die Untersuchung des normalen Magensafts abgeschlossen war, wurde einer der Hunde mit Anilin vergiftet, der andere mit Pyrogallussäure; der dritte wurde wiederholt und ausgiebig zu Ader gelassen. Als die Blutzusammensetzung (es wurden die Erythrozytenzahl und der Hämoglobinwert bestimmt) stark verändert zu sein schien, wurde erneut der pHWert des Blutes registriert und anschließend eine neue Analysenserie des Magensafts in

\footnotetext{
${ }^{57}$ Schtscherbakow, A. I., Über die Bedingungen der Entwicklung des runden Magengeschwürs (Ulcus ventriculi chronicum rotundum), S. 403.

${ }^{58}$ Schtscherbakow, A. I., Über die Bedingungen der Entwicklung des runden Magengeschwürs (Ulcus ventriculi chronicum rotundum), S. 405.
} 
denselben Zeitintervallen durchgeführt - unter strikter Einhaltung aller Bedingungen, unter denen die erste Versuchsreihe durchgeführt worden war. Das Ziel dieser Experimente bestand in der Untersuchung der Veränderungen des Gehalts von Salzsäure im Magensaft unter Einwirkung der gestörten Blutzusammensetzung. Die Ergebnisse der Versuche zeigten, dass bei einer ausgeprägten Anämie eine Erhöhung der Salzsäurekonzentration zu verzeichnen ist, ebenso eine wesentliche Verstärkung der Magensekretion. Schtscherbakow hatte genau dies erwartet, denn es war eine Vielzahl von klinischen Fällen bekannt, bei der eine verstärkte Magensekretion und ein hyperazider Zustand mit einer Anämie (Chlorose) einhergingen.

Die letzte Versuchsserie war von herausragender Bedeutung für die Aufklärung der Ätiologie der Ulkuskrankheit, denn hierbei ergab sich, dass es Faktoren gibt, die gleichzeitig beide zentralen Elemente innerhalb der kausalen Pathogenese der Erkrankung beeinflussen: die verminderte Resistenz des Magengewebes mit lokalen Wandschädigungen und der erhöhte Säuregehalt des Magensafts. „Die Veränderung der Blutzusammensetzung“, notiert Schtscherbakow, „die eine anomale Ernährung des Gewebes resp. eine geschwächte Resistenz gegen seine schädlichen Sekrete bewirkt, kann tatsächlich oft mit einer erhöhten Magensaftazidität verbunden sein. Das Zusammenspiel dieser beiden Faktoren, von denen jeder den anderen bedingt, ist es, das die günstigsten Bedingungen für den fortschreitenden Verlauf der Ulzeration schafft, obwohl jeder von den genannten Faktoren allein kein absolutes ätiologisches Moment des Geschwürs darstellt." 59

Von Interesse war für Schtscherbakow auch die Frage nach der Wirkung der in den Magen eingebrachten Salzsäure unter den Bedingungen einer Anämie.

Der Einfluss der Salzsäure auf die Magenwand bei einer Anämie wurde wie folgt untersucht: Dem Magen wurde durch ein Fistelrohr die verdünnte (4,5\%ige) und auf Körpertemperatur erwärmte Salzsäure zugeführt. Binnen einiger Tage nach dem Beginn der HCI-Verabreichung gingen die Hunde ein. Bei der Autopsie der Tiere ergab sich, dass bei der Anilinvergiftung und mehreren Aderlässen im Magen Veränderungen an der Wand zu beobachten sind, die für das perforierende Ulcus ventriculi rotundum kennzeichnend sind. Bei den Pyrogallolvergiftungen waren diese Veränderungen weniger deutlich ausgeprägt.

Schtscherbakow berücksichtigte alle Erkenntnisse seiner Zeit und die Ergebnisse seiner eigenen Versuche. Auf dieser Grundlage postulierte er als ,,im Hintergrund“ wirksame „finale“ Ursache der Entstehung und Entwicklung des Geschwürs Folgendes: „Das Zusammenwirken einiger Faktoren, die die Entwicklung einer Ulzeration in einem ätiologischen Moment, nämlich einem anämischen Zustand, fördern, scheint für die Lehre der Ursachen des Ulcus ventriculi rotundum besonders wichtig zu sein: Die Veränderung der Blutzusammensetzung, die eine anomale Gewebeernährung bedingt resp. die Fähig-

\footnotetext{
${ }^{59}$ Schtscherbakow, A. I., Über die Bedingungen der Entwicklung des runden Magengeschwürs (Ulcus ventriculi chronicum rotundum), S. 459.
} 
keit des Gewebes, den schädlichen Einflüssen entgegenzuwirken, kann oft mit einem wichtigen Faktor verbunden sein, der die Ulzeration fördert, und dies ist die verstärkte Magensaftsekretion; die Kombination von beiden, sich gegenseitig unterstützenden Faktoren schafft besonders günstige Bedingungen für eine Geschwürentwicklung, die dazu neigt, mehr oder weniger schnell fortzuschreiten und unseren therapeutischen Maßnahmen in keiner Weise nachzugeben."60

Daraus kann man folgern, dass Schtscherbakow auf experimentellem Weg nachwies, dass primäre nicht spezifische Ulzerationen der Magenschleimhaut und das Ulcus ventriculi nicht identisch sind und dass die Schädigung der Kontinuität der Magenwand nur einer der Faktoren ist, der unter bestimmten Bedingungen zur Entstehung und fortschreitenden Entwicklung der Ulkuskrankheit führen kann. Der zweite wichtige Faktor der Pathogenese des Geschwürs ist die erhöhte Magensaftazidität. Im Zusammenspiel dieser Faktoren zu einem bestimmten Zeitpunkt und in einer bestimmten Reihenfolge liegt das Wesen der Pathogenese des Geschwürs oder, wie Schtscherbakow festhält, die „causa proxima ulceris ventriculi rotundi“.

Die ultimative Ursache der Geschwürbildung sah er in der Veränderung der Trophik des Magengewebes und der daraus resultierenden geschwächten Geweberesistenz. Die Zirkulation in bestimmten Bereichen wurde gestört, es entstand die Ulzeration, und die Magensaftazidität erhöhte sich. Die Koinzidenz von diesen zwei Momenten führte zur Entwicklung des runden Geschwürs, das den Klinikern als spezifische nosologische Einheit bekannt ist.

Mit Rücksicht auf alles Dargelegte können wir mit vollem Recht behaupten, dass die wissenschaftlich begründete ganzheitliche Theorie der Ätiologie und der Pathogenese der Ulkuskrankheit zum ersten Mal in der Geschichte der internationalen Wissenschaft von Alexej Iwanowitsch Schtscherbakow formuliert worden ist.

Schtscherbakows Werke wurden von seinen Zeitgenossen gewürdigt; dies kann man von seinen Nachfahren nicht behaupten. Bereits zu Beginn des 20. Jahrhunderts wurden die Ergebnisse seines fundamentalen Werks von seinen Landsleuten nicht mehr wahrgenommen. Autoren außerhalb Russlands kamen erst deutlich später zu ähnlichen Schlussfolgerungen. Der gern zitierte Ausruf von K. Schwarz, „Ohne Säure kein Geschwür“, erscholl in den 1920er Jahren. Heutzutage steht A. Shay hoch im Ansehen; er hat die Idee von der Disparität der aggressiven Faktoren aus der Umwelt und der Schutzfaktoren der Schleimhaut im Jahre 1959 (!!!) geäußert. In Russland, so S. S. Judin, schätzten die Chirurgen die Bedeutung des Säurefaktors, weil sie zusammen mit den Kranken einen Weg bitterer Fehler und Enttäuschungen zurückgelegt hatten.

Es muss leider festgestellt werden, dass die „krankhafte Vergesslichkeit“ bezüglich der Leistungen der russischen Wissenschaftler im Falle von Schtscherbakow eine lange Geschichte und eine nachhaltige Tendenz hat. Wir möchten dazu nur einige Beispiele

\footnotetext{
${ }^{60}$ Schtscherbakow, A. I., Über die Bedingungen der Entwicklung des runden Magengeschwürs (Ulcus ventriculi chronicum rotundum), S. 430-434.
} 
anführen. 1909 hielt auf dem 19. Kongress der Russischen Chirurgen, auf dem zum ersten Mal die Forderung nach dem auf der Pathogenese basierenden Vorgehen bei der chirurgischen Behandlung des Magengeschwürs erhoben wurde, der damals bekannte Chirurg K. M. Ssapeshko einen Vortrag mit dem Titel „Chirurgische Behandlung eines komplikationsfreien runden Magengeschwürs“. Er berichtete über seine Versuche an Kaninchen und Hunden, bei denen er die Pathogenese des Magengeschwürs simulierte, ohne sich auf die Arbeiten von Schtscherbakow zu beziehen. In der Diskussion zum Beitrag von Ssapeshko sagte I. K. Sspisharny: „Was die Pathogenese des runden Geschwürs betrifft, so muss ich sagen, dass Versuche, die mit den Versuchen von Prof. Ssapeshko identisch sind, vor 20 Jahren von Dr. Schtscherbakow im Labor von Prof. Focht durchgeführt wurden. Schon damals ist er zur Schlussfolgerung gekommen, dass den größten Einfluss auf die Entstehung des Magengeschwürs die Veränderungen der Gefäße haben.“ Der Opponent der Doktorarbeit von Schtscherbakow, Prof. W. D. Scherwinski, betonte, dass dessen Modell eine der charakteristischen Eigenschaften des Geschwürs erklärt, nämlich seinen chronischen Verlauf: „Die Chronizität des Verlaufs - darin besteht das Wesen. Auch dies wurde teilweise experimentell geklärt; Prof. Schtscherbakow wies daraufhin, dass hier die Anämie eine Rolle spielt.“

Und noch ein markantes Beispiel, das veranschaulicht, wie verbreitet das Unwissen über die Geschichte der einheimischen Wissenschaft war: Auf dem 19. Chirurgenkongress stellte O. W. Nikolajew fest: „Indem wir uns der entscheidenden klinischen Schlussfolgerungen enthalten und im Rahmen eines reinen Experiments bleiben, möchten wir die These von George Galperin aus Chicago [...] hervorheben, die besagt, dass man an das Problem der Pathogenese des Geschwürs von zwei Seiten herangehen kann - erstens, durch die Untersuchung der Ursachen für die Entstehung der Erosion und, zweitens, durch die Untersuchung der Entstehungsbedingungen eines chronischen Geschwürs aus der Erosion."

Auf der 1933 in Moskau abgehaltenen Konferenz über die Probleme der Ulkuskrankheit wurden der Ätiologie und Pathogenese dieser Erkrankung mehrere Berichte gewidmet. Bekannte Wissenschaftler ergriffen das Wort: R. A. Lurija, W. T. Talalajew, F. M. Plotkin u.a.m. Aber in keinem Bericht gab es eine Bezugnahme auf die Studien von Schtscherbakow, obwohl die Ergebnisse mehrerer Untersuchungen, die auf dieser Tagung erörtert wurden, in puncto Qualität die Arbeiten von Schtscherbakow nicht erreichten.

Leider ist die Geschichte der russischen Wissenschaft reich an Phänomenen einer solchen „Vergesslichkeit“. Den Historikern der russischen Wissenschaft steht es noch bevor zu verstehen, was die Kliniker und ihre Patienten dieser Verlust an Kontinuität gekostet hat. In diesem Sinne stellt die Geschichte der Behandlung der Ulkuskrankheit keine Ausnahme dar.

Schtscherbakow fasste die Ergebnisse seiner Versuche zusammen und kam zu folgendem allgemeinen Resümee: „Die Entstehung des Geschwürs ist in den meisten Fällen mit der Störung der Blutversorgung in einem bestimmtem Bereich der Magenwand verbunden [...]. Die Störung der Unversehrtheit der Magenwand stellt noch kein 
Geschwür dar, sie verheilt [...] als glatte Narbe innerhalb einer sehr kurzen Frist. Damit sich eine Läsion zu einem Ulcus ventriculi entwickeln kann, ist die Wirkung bekannter Faktoren erforderlich; als solche kann man auf der Grundlage klinischer Beobachtungen und einiger Versuche die Erhöhung der Magensaftazidität und die Resistenzschwäche des Gewebes erkennen." 61

Schtscherbakow stellt die Pathogenese des Geschwürs im Allgemeinen wie folgt dar: Der hyperazide Mageninhalt wirkt aggressiv auf die Schleimhaut ein; deren Schutzmechanismen sind als Ergebnis eines Komplexes von lokalen (Störung der Blutversorgung) und allgemeinen (Reizeinfluss des Zentralnervensystems) Faktoren geschwächt. Der ulzeröse Defekt, der sich infolge der Störung der Trophik der Magenwand herausbildet, schreitet unter dem Einfluss der erhöhten Magensaftazidität chronisch fort; infolgedessen entsteht die chronische Ulzeration, die durch eine Reihe von klinischen Symptomen in Erscheinung tritt - die eigentliche Ulkuskrankheit. Unter anderem schreibt Schtscherbakow: „Von den Formen der erhöhten periodischen Sekretion sind strikt die Fälle zu trennen, bei denen die funktionelle Störung erst bei einer gewissen Reizung des Magens (durch gewöhnliche oder ungewöhnliche auslösende Reize) und bei denen die erhöhte Magensaftabsonderung ohne jeglichen äußeren Anlass (unter dem Einfluss innerer Reize, die bei Erkrankungen des Nervensystems auf zentrale Bereiche des Nervenapparates wirken, der mit den sekretorischen Magenorganen verbunden ist) eintritt. “62

Unseres Erachtens können wir mit Überzeugung festhalten, dass eine vollständige Theorie der Ätiologie und Pathogenese der Ulkuskrankheit zum ersten Mal von einem russischen Wissenschaftler formuliert worden ist.

\footnotetext{
${ }^{61}$ Schtscherbakow A. I., Über die Bedingungen der Entwicklung des runden Magengeschwürs (Ulcus ventriculi chronicum rotundum), S. 317.

${ }^{62}$ Schtscherbakow A. I., Über die Bedingungen der Entwicklung des runden Magengeschwürs (Ulcus ventriculi chronicum rotundum), S. 317.
}

Open Access Dieses Kapitel wird unter der Creative Commons Namensnennung 4.0 International Lizenz (http://creativecommons.org/licenses/by/4.0/deed.de) veröffentlicht, welche die Nutzung, Vervielfältigung, Bearbeitung, Verbreitung und Wiedergabe in jeglichem Medium und Format erlaubt, sofern Sie den/die ursprünglichen Autor(en) und die Quelle ordnungsgemäß nennen, einen Link zur Creative Commons Lizenz beifügen und angeben, ob Änderungen vorgenommen wurden.

Die in diesem Kapitel enthaltenen Bilder und sonstiges Drittmaterial unterliegen ebenfalls der genannten Creative Commons Lizenz, sofern sich aus der Abbildungslegende nichts anderes ergibt. Sofern das betreffende Material nicht unter der genannten Creative Commons Lizenz steht und die betreffende Handlung nicht nach gesetzlichen Vorschriften erlaubt ist, ist für die oben aufgeführten Weiterverwendungen des Materials die Einwilligung des jeweiligen Rechteinhabers einzuholen.

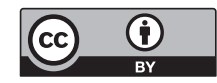

